\title{
Equivalence Principle Violations and Couplings of a Light Dilaton
}

\author{
Thibault Damour ${ }^{a}$ and John F. Donoghue ${ }^{a, b}$, \\ ${ }^{a}$ Institut des Hautes Études Scientifiques \\ Bures sur Yvette, F-91440, France \\ and \\ ${ }^{b}$ Department of Physics \\ University of Massachusetts \\ Amherst, MA 01003, USA
}

October 31, 2018

\begin{abstract}
We consider possible violations of the equivalence principle through the exchange of a light 'dilaton-like' scalar field. Using recent work on the quark-mass dependence of nuclear binding, we find that the dilaton-quark-mass coupling induces significant equivalence-principleviolating effects varying like the inverse cubic root of the atomic number $-A^{-1 / 3}$. We provide a general parameterization of the scalar couplings, but argue that two parameters are likely to dominate the equivalence-principle phenomenology. We indicate the implications of this framework for comparing the sensitivities of current and planned experimental tests of the equivalence principle.
\end{abstract}




\section{Introduction}

At the heart of the theory of General Relativity is Einstein's Equivalence Principle (EP). The weak Equivalence Principle predicts the composition independence of the accelerations of test masses in a gravitational field. This has been probed at a present sensitivity of

$$
\frac{\Delta a}{a} \sim 10^{-13}
$$

in innovative and difficult experiments [1, 2]. Further tests of this principle remain important and relevant for new physics [3, 4]. We are fortunate that there are several initiatives to push the sensitivity several orders of magnitude further using new space-based experiments such as MICROSCOPE [5], the Galileo Galilei project [6] and STEP [7] as well as new types of experiments using cold atoms [8, 9] and sub-orbital rockets [10].

One possible source of EP violation is a very light 1 scalar field with a coupling to matter that is weaker than gravitational strength. We will refer to these generically as 'dilatons', although they may have origins other than string theory or models involving dilation symmetry. As will become clear below, we will phenomenologically define a 'dilaton' as a scalar field $\phi$ whose couplings to matter effectively introduce a $\phi$ dependence in the basic dimensionless constants of Nature (such as the fine-structure constant etc.). String theory may have such scalars in the low energy limit ( string dilaton, moduli), and these can naturally lead to EP violation at a sizeable level [1, 12, 13, 14, 15. Likewise, theories of quintessence predict a light scalar, as do theories with continuously varying coupling constants as well as some theories of dark matter. While scalars lead to an attractive interaction, like usual gravity, they do not couple universally to all forms of energy in the same way as in general relativity. Thus we expect differences in the forces for different elements.

Additionally, independently of any specific theoretical model one might argue (along the 'anthropic' approach to the issue of a possibly extremely vast 'multiverse' of cosmological and/or string backgrounds) that: (i) the 'Equivalence Principle' is not a fundamental symmetry principle of Nature (e.g. it is 'violated' in any theory containing very light scalars); (ii) the level $\eta \sim \Delta a / a$ of EP violation can be expected to vary, quasi randomly, within some range of order unity, over the full multiverse of possible (cosmological and/or theoretical) backgrounds; (iii) as there is probably a maximal level

\footnotetext{
${ }^{1}$ We will generally assume in the following that the scalar field we consider is essentially massless on the scales that we discuss.
} 
of EP violation, say $0<\eta_{*} \ll 1$, which is compatible with the development of life (and of physicists worrying about the EP), one should a priori expect to observe, in our local environment, an EP violation $\eta$ of order of $\eta_{*}$. It is a challenge to give a precise estimate (or at least upper bound) of $\eta_{*}$. We note, however, that this is a scientifically rather well-posed challenge. For instance, one of the necessary conditions for the existence of life is the existence of solar-like planetary systems stable over billions of years. A sufficiently large $\eta \neq 0$ will jeopardize this stability, notably under the influence of external, passing stars. The current very small level of EP violation ensures that stars passing at a distance $D$ disturb the inner dynamics of the solar system only through tidal effects that decrease like $D^{-3}$. An EP violation $\eta$ would increase this disturbing effect to a level $\propto \eta R^{-2}$. It is also a well-posed question to determine the level $\eta$ which would destabilize the solar system through internal EP-violating gravitational effects.

Independently of these various motivations, our work here will discuss the general type of composition-dependence of EP violation that is entailed by the existence of a light dilaton-like field. The theoretical challenge is to connect the basic couplings of the dilaton Lagrangian to the properties of real atomic systems.

Our work starts in Section 2 with a review of EP violations, and a general parameterization of possible dilaton couplings, Eq. (12). Section 3 connects dilaton coupling parameters with the other couplings of the Standard Model, which is preparation for understanding the effects of the dilaton couplings. Section 4 is our analysis of the effects in nuclear binding, while Section 5 is a summary of the effects within individual nucleons, and Section 6 describes electromagnetic effects. In Section 7, we collect the results of the previous sections and give a complete treatment of the phenomenology of equivalence principle violations, including comparisons with existing experiments. Section 8 provides a guide to experimental sensitivities for existing and future experiments. Experimenters who are willing to forgo the theoretical development of Section 3-6 can go directly to Section 7-8 or can consult our shorter paper [16] in which we have collected our most phenomenologically useful results. In particular, Section 7.3 contains what is probably the most useful parameterization of our results and Section 7.4 discusses the present experimental constraints. Section 9 is a brief summary. 


\section{Formalism}

\section{$2.1 \quad$ EP violation}

Let us start by recalling that a massless dilaton $\phi$ modifies the Newtonian interaction between a mass $A$ and a mass $B$, into the form (see, e.g. [14])

$$
V=-G \frac{m_{A} m_{B}}{r_{A B}}\left(1+\alpha_{A} \alpha_{B}\right) .
$$

If the dilaton mass is important the second term includes an extra exponential factor $\exp \left(-m_{\phi} r_{A B}\right)$. In this interaction potential, the scalar coupling to matter is measured by the dimensionless factor

$$
\alpha_{A}=\frac{1}{\kappa^{2} m_{A}} \frac{\partial\left[\kappa m_{A}(\phi)\right]}{\partial \phi} .
$$

Here, $\kappa \equiv \sqrt{4 \pi G}$ is the inverse of the Planck mass 22 so that the product $\kappa m_{A}$ is dimensionless. This ensures that this definition of $\alpha_{A}$ is valid in any choice of units, even if these units are such that $\kappa$ depends on $\phi$ (as in the so-called 'string frame'). In the following, we shall generally assume that we work in the 'Einstein frame' where the (bare) Newton constant $G$ is independent of $\phi$. The above expression for the dimensionless scalar coupling $\alpha_{A}$ has been written in terms of a canonically normalized scalar field, with kinetic term [using the signature $(+,-,-,-)$ ]

$$
\mathcal{L}_{\phi}=\frac{1}{2}(\partial \phi)^{2}+\cdots
$$

Evidently, a small mass term for the dilaton can readily be added if desired. It can also be convenient to work with the dimensionless scalar field

$$
\varphi \equiv \kappa \phi,
$$

whose kinetic term is related to the Einstein-Hilbert action via

$$
-\frac{1}{16 \pi G}\left(R-2(\partial \varphi)^{2}\right)
$$

When using $\varphi$ the definition of the dimensionless scalar coupling reads

$$
\alpha_{A}=\frac{\partial \ln \left[\kappa m_{A}(\varphi)\right]}{\partial \varphi} .
$$

\footnotetext{
${ }^{2}$ We use units such that $c=1=\hbar$.
} 
In terms of the $\alpha_{A}$ 's, the violation of the (weak) $\mathrm{EP}$, i.e. the fractional difference between the accelerations of two bodies $A$ and $B$ falling in the gravitational field generated by an external body $E$, reads

$$
\left(\frac{\Delta a}{a}\right)_{A B} \equiv 2 \frac{a_{A}-a_{B}}{a_{A}+a_{B}}=\frac{\left(\alpha_{A}-\alpha_{B}\right) \alpha_{E}}{1+\frac{1}{2}\left(\alpha_{A}+\alpha_{B}\right) \alpha_{E}} \simeq\left(\alpha_{A}-\alpha_{B}\right) \alpha_{E} .
$$

In the last (approximate) equation we have assumed that the $\alpha$ 's are small, so that one can neglect the term $\frac{1}{2}\left(\alpha_{A}+\alpha_{B}\right) \alpha_{E}$ in the denominator.

Our aim here is to provide a general analysis of the possible EP violations in experiments comparing the free fall accelerations of atoms (and/or nuclei). Most of the effort needed for such an analysis is now understood [12, 13, 15, 17, and we will use it below. However, one aspect of this analysis has been far less well-studied and understood, namely the contribution to $\mathrm{EP}$ violation coming from the possible $\phi$-dependence of the nuclear binding energy. The aim of this paper will mainly be to assess the form of this contribution, coming from the quark mass contribution to nuclear binding 3 . Actually, our conclusion will be that this contribution is, possibly in competition with Coulomb-binding effects, likely to dominate the atom-dependence of the EP violation signal (8).

To motivate our general analysis, let us start by noting that the mass of an atom can be decomposed as

$$
m\left(\operatorname{Atom}_{A}\right)=m_{A}=m_{A}^{\text {rest mass }}+E^{\text {binding }}
$$

where

$$
m_{A}^{\text {rest mass }}=Z m_{p}+N m_{n}+Z m_{e}
$$

is the rest-mass contribution to the mass of an atom $(Z$ denoting the atomic number and $N$ the number of neutrons), and where $E^{\text {binding }}$ is the binding energy of the atom, which is dominated by the binding energy of the nucleus. $E^{\text {binding }} \equiv E_{3}+E_{1}$ is the sum of a strong interaction contribution, say $E_{3}$, and of an electromagnetic one, say $E_{1}$ (which is dominated by the electromagnetic effect within the nucleus). The indices 3 and 1 are used here as reminders of the gauge groups underlying the considered interactions: namely, $S U(3)$ and $U(1)$. Note that the index $A$ in $m_{A}$ is used here (like in the definition of the scalar coupling $\alpha_{A}$ ) as a label for distinguishing several different atoms. It should not be confused with the mass number (or nucleon number) $A \equiv Z+N$ which we shall use below.

\footnotetext{
${ }^{3}$ Damour [3] and Dent [17] have highlighted this need for the study of the nuclear binding energies.
} 


\subsection{The general dilaton Lagrangian}

The basic organizing principle that we shall use in our discussion is to keep track of the effect of all the possible $\phi$ modifications of the terms entering the effective action describing physics at the scale of nuclei in their ground states. We have in mind here an energy scale $\mu \sim 1 \mathrm{GeV}$. At such a scale, one has integrated out not only the effect of weak interactions, but also the heavy quarks $c, b$ and $t$. The issue of the possible $\phi$ sensitivity of effects linked to the strange quark $s$ is more delicate. In the Appendix we argue that the possible EP violations linked to the $\phi$ couplings to $s$ are expected to be quite small. In the bulk of the text we shall therefore ignore $s$ (assuming that its effect is taken into account by changing some of the quantities we discuss, notably the QCD energy scale $\Lambda_{3}$ ).

In this approximation, we are therefore talking about an effective action containing, as real particles, the electron $e$, the $u$ quark, and the $d$ quark, with interactions mediated by the electromagnetic $\left(A_{\mu}\right)$ and gluonic $\left(A_{\mu}^{A}\right)$ fields. [Here we shall use a rescaled $U(1)$ gauge potential, which incorporates the electron charge $e$, but an unrescaled gluonic field, which does not incorporate the $S U(3)$ gauge coupling $g_{3}$.] Then each of the five terms in this effective action, say

$$
\mathcal{L}_{\mathrm{eff}}=-\frac{1}{4 e^{2}} F_{\mu \nu} F^{\mu \nu}-\frac{1}{4} F_{\mu \nu}^{A} F^{A \mu \nu}+\sum_{i=e, u, d}\left[i \bar{\psi}_{i} \not D\left(A, g_{3} A^{A}\right) \psi_{i}-m_{i} \bar{\psi}_{i} \psi_{i}\right]
$$

(where $D(A)$ denotes the Dirac operator coupled to the gauge field(s) $A$ ) can couple to $\varphi=\kappa \phi$ with a (dimensionless) coefficient. [We assume that we work in the Einstein frame, with the gravity and $\phi$ kinetic terms displayed above.] This introduces five dimensionless dilaton coupling coefficients, say $d_{e}, d_{g}$ for the couplings to the electromagnetic and gluonic field terms, and $d_{m_{e}}, d_{m_{u}}, d_{m_{d}}$ for the couplings to the fermionic mass terms. We shall normalize these five dimensionless dilaton coupling coefficients $d_{e}, d_{g}, d_{m_{e}}, d_{m_{u}}, d_{m_{d}}$ so that they correspond (when considering the linear couplings to $\phi$ ) to the following interaction terms

$$
\mathcal{L}_{\text {int } \phi}=\kappa \phi\left[+\frac{d_{e}}{4 e^{2}} F_{\mu \nu} F^{\mu \nu}-\frac{d_{g} \beta_{3}}{2 g_{3}} F_{\mu \nu}^{A} F^{A \mu \nu}-\sum_{i=e, u, d}\left(d_{m_{i}}+\gamma_{m_{i}} d_{g}\right) m_{i} \bar{\psi}_{i} \psi_{i}\right]
$$

\footnotetext{
${ }^{4}$ We are using here the fact that a $\phi$-dependent coupling to the kinetic term of a fermion, $f(\phi) \bar{\psi} i \not D \psi$, can be absorbed in a suitable $\phi$-dependent rescaling of $\psi$.
} 
We shall explain below the notation and our choice of normalization for these interaction terms.

There are two equivalent ways of thinking about the computation of the scalar-matter coupling $\alpha_{A}$, Eq. (3). One way is to think that it is given by the matrix element (in the quantum state of an atom) of the operator in the quantum Hamiltonian (associated to the interaction Lagrangian above) which is linear in $\phi$. A second way is to think that it is obtained by the chain rule as

$$
\alpha_{A}=\frac{\partial \ln \left[\kappa m_{A}(\varphi)\right]}{\partial \varphi}=\sum_{a} \frac{\partial \ln \left[\kappa m_{A}\left(k_{a}\right)\right]}{\partial k_{a}} \frac{\partial k_{a}}{\partial \varphi} .
$$

where $\kappa m_{A}\left(k_{a}\right)$ is the expression of the dimensionless mass ratio $\kappa m_{A}=$ $m_{A} / m_{\text {Planck }}$ as a function of the dimensionless coupling constants of Nature, say $k_{a}=k_{1}, k_{2}, \ldots, k_{20}$, entering the Standard Model. Actually, because of the limited number of terms entering the relevant low-energy action (11), there are only five relevant dimensionless constants of Nature $k_{a}$ corresponding to the five terms in (11). As we shall see in detail below, the five terms in the interaction terms (12) precisely correspond to introducing a $\phi$ dependence in the five following dimensionless constants of Nature,

$$
\alpha, \kappa \Lambda_{3}, \kappa m_{e}, \kappa m_{u}, \kappa m_{d}
$$

where $\alpha=e^{2} /(4 \pi)$ is the fine-structure constant, $\Lambda_{3}$ the QCD energy scale, $m_{e}$ the electron (pole) mass, and where $m_{u}$ and $m_{d}$ denote some renormalization-group-invariant measures of the light quark masses (say, the $\mu$-running masses taken at the multiple of $\Lambda_{3}$ which is equal to $1 \mathrm{GeV}$ ).

In the next Section we shall relate our normalisation of the five dimensionless dilaton coupling parameters $d_{a}$ entering (12) to the constants (14), and explain in more detail the dependence of the mass of an atom on the five constants (14), and thereby on the five dilaton parameters $d_{a}$.

\section{Relation between the dilaton coupling parame- ters $d_{a}$ and the 'constants of Nature'.}

By comparing the $\phi$-interaction Lagrangian (12) to the other terms in the effective action (11), we see that the meaning of the dilaton coupling coefficients $d_{a}=d_{e}, d_{g}, d_{m_{e}}, d_{m_{u}}, d_{m_{d}}$ seems clear for four of them. [Actually, we shall see below that the meaning of the quark-mass couplings $d_{m_{i}}$ is more subtle, because of the renormalization group running of the quark masses, 
which is associated with the $\gamma_{m_{i}} d_{g}$ term in (12).] First, the coupling $d_{e}$ to the electromagnetic field modifies the Maxwell action according to

$$
\mathcal{L}_{E M}=-\frac{1-d_{e} \kappa \phi}{4 e^{2}} F_{\mu \nu} F^{\mu \nu} \simeq-\frac{1}{4\left(1+d_{e} \kappa \phi\right) e^{2}} F_{\mu \nu} F^{\mu \nu}
$$

where the last equality is valid at the linear level in $\kappa \phi$ (which is the level at which we define the dilaton couplings here). As we work with a rescaled electromagnetic field ( $A^{\text {here }}=e A^{\text {usual }}$ ), the only location where the electric charge occurs in the Lagrangian is the one explicitly shown above. This allows the dilaton field to be absorbed into the following $\phi$ dependence of the fine-structure constant

$$
\alpha(\phi)=\left(1+d_{e} \kappa \phi\right) \alpha=\left(1+d_{e} \varphi\right) \alpha .
$$

Second, comparing (12) to the mass terms of the electron and the light quarks, we see that our normalization is such that $d_{m_{e}}, d_{m_{u}}, d_{m_{d}}$ introduce the following $\phi$ dependence of the $e, u$ and $d$ masses:

$$
m_{i}(\phi)=\left(1+d_{m_{i}} \kappa \phi\right) m_{i}=\left(1+d_{m_{i}} \varphi\right) m_{i},(i=e, u, d) .
$$

On the other hand, the terms in (12) that depend on our 'dilaton-gluon' coupling $d_{g}$ call for a more subtle explanation. The choice of these coupling terms is such that the coefficient of $d_{g}$ is invariant under the renormalizationgroup (RG). As the coefficient of $d_{m_{i}}$ (i.e. the mass term $m_{i} \bar{\psi}_{i} \psi_{i}$ ) is also, separately, RG-invariant, our choice of normalization of the coefficients in (12) gives a RG-invariant meaning to both $d_{g}$ and the $d_{m_{i}}$ 's 5

\subsection{Connection with the QCD trace anomaly}

The phenomenological consequences (for the scalar coupling to hadrons) of the RG-invariant nature of the couplings in (12) can be seen in two (equivalent) ways. One way (which was used by [18] and [15]) consists in remarking that the definition of the $d_{g}$-dependent terms in (12) is such that they couple $\phi$ to the anomalous part of the trace of the gluon stress-energy tensor, namely

$$
\mathcal{L}_{g \phi}=-d_{g} \kappa \phi T_{g}^{\text {anom }}
$$

\footnotetext{
${ }^{5}$ We are here talking about invariance under the QCD-driven running of the QCD gauge coupling $g_{3}$, and of the masses of fermions coupled to QCD. In view of the smallness of the electromagnetic coupling $\alpha \simeq 1 / 137 \ll \alpha_{3}$, we are neglecting the RG-running driven by electromagnetic effects. If one wanted to take it into account, one should add to (12) additional terms linked to the QED trace anomaly.
} 
where [19]

$$
T_{g}^{\mathrm{anom}}=\left[\frac{\beta_{3}}{2 g_{3}} F_{\mu \nu}^{A} F^{A \mu \nu}+\gamma_{m} \sum_{i} m_{i} \bar{\psi}_{i} \psi_{i}\right]_{\mu}
$$

Here, $\beta_{3}\left(g_{3}\right)=\mu \partial g_{3} / \partial \mu$ denotes the $\beta$ function for the running of the QCD coupling $g_{3}$ with the (Wilsonian) sliding energy scale $\mu, \gamma_{m}\left(g_{3}\right)=$ $-\mu \partial \ln m / \partial \mu$ (with a minus sign on the r.h.s.) is the (universal) anomalous dimension giving the energy-running of the masses of the QCD-coupled fermions, and the subscript $\mu$ at the end indicates that the operator on the r.h.s. must be renormalized at the running scale $\mu$. We recall that, classically, the trace of the gluonic stress-energy tensor vanishes (because of the conformal invariance of the Yang-Mills action), but that quantum effects linked to the necessity of regularizing the UV infinities in the product of gluon field strengths at the same spacetime point $x$ introduce the (finite) 'conformal anomaly' (19) [19]. Then, by using the quantum version of the virial theorem6, one can see [18, 15] that the coupling (18) means that $d_{g}$ measures the coupling of $\phi$ to the part of the total mass-energy of the considered hadron which is due to the (renormalized) gluonic field energy, say $M_{g}$ (where $M_{g}$ can be defined by subtracting from the total mass both the nonanomalous mass-term contributions $\left\langle\sum_{i} m_{i} \bar{\psi}_{i} \psi_{i}\right\rangle$, and the electromagnetic one).

\subsection{Renormalization group analysis}

A second way of discussing the consequences (for the scalar coupling to hadrons) of our normalization of couplings in (12) is phenomenologically illuminating. It consists in noting that our RG-invariant definitions are equivalent to very simple consequences for the $\phi$ dependences of both the QCD mass scale $\Lambda_{3}$, and the values of the quark masses at the scale $\mu=$ $\Lambda_{3}$. [Note that both $\Lambda_{3}$ and $m_{i}\left(\Lambda_{3}\right)$ are RG-invariantly defined quantities.] Let us start by defining the QCD mass scale $\Lambda_{3}$ as being the mass scale at which the running QCD coupling $g_{3}(\mu)$ reaches some fixed, reference dimensionless number of order unity, say $g_{*}=2.5$. [This numerical value, which corresponds to $\alpha_{*}=g_{*}^{2} /(4 \pi)=0.5$, is approximately reached when the running scale $\mu \simeq 1 \mathrm{GeV}$ (see, e.g., the figure giving $\alpha_{s}(\mu)$ in the QCD review in [20]).] This definition of $\Lambda_{3}$ can be re-expressed in terms of the value $g_{c} \equiv g_{3}\left(\Lambda_{c}\right)$ of $g_{3}$ at some high-energy 'cut-off' scale $\Lambda_{c}$ (which could

\footnotetext{
${ }^{6}$ We recall that this theorem says that the space integral of the spatial components of the total stress-energy tensor $T_{\text {tot }}^{\mu \nu}=T_{g}^{\mu \nu}+T_{E M}^{\mu \nu}+T_{\text {matter }}^{\mu \nu}$ vanishes in an equilibrium bound state.
} 
be the Planck scale, or the string scale) by integrating the $\beta$ equation giving the running of $g_{3}, d \ln \mu=d g_{3} / \beta_{3}\left(g_{3}\right)$, so that:

$$
\ln \Lambda_{3}\left(\Lambda_{c}, g_{c}\right)=\ln \Lambda_{c}-\int_{g_{*}}^{g_{c}} \frac{d g_{3}}{\beta_{3}\left(g_{3}\right)}
$$

The expression (20) defines $\Lambda_{3}$ as a function of $\Lambda_{c}$ and $g_{c}$. If we assume for simplicity that the chosen cut-off $\Lambda_{c}$ does not depend (in the Einstein frame) on $\phi$, the result (20) shows that $\Lambda_{3}$ will inherit a $\phi$ dependence from any eventual $\phi$ dependence of $g_{c}$ according to (denoting $\beta_{c} \equiv \beta_{3}\left(g_{c}\right)$ )

$$
\frac{\partial \ln \Lambda_{3}}{\partial \varphi}=-\frac{g_{c}}{\beta_{c}} \frac{\partial \ln g_{c}}{\partial \varphi}
$$

Similarly, the integration of the RG equation for a running fermionic mass $m_{i}$, namely $d \ln m_{i}=-d g_{3} \gamma_{m}\left(g_{3}\right) / \beta_{3}\left(g_{3}\right)$ yields the following expression for the value of $m_{i}$ at the QCD scale, $\ln m_{i}\left(\Lambda_{3}\right)$ :

$$
\ln m_{i}\left(\Lambda_{3}\right)=\ln m_{i}\left(\Lambda_{c}\right)+\int_{g_{*}}^{g_{c}} \frac{\gamma_{m}\left(g_{3}\right)}{\beta_{3}\left(g_{3}\right)} d g_{3}
$$

Differentiating this result w.r.t. $\varphi$ then shows that the logarithmic derivative of $m_{i}\left(\Lambda_{3}\right)$ w.r.t. $\varphi$ is the sum of two separate contributions, namely (denoting $\gamma_{c} \equiv \gamma_{m}\left(g_{c}\right)$ )

$$
\frac{\partial \ln m_{i}\left(\Lambda_{3}\right)}{\partial \varphi}=\frac{\partial \ln m_{i}\left(\Lambda_{c}\right)}{\partial \varphi}+\frac{g_{c} \gamma_{c}}{\beta_{c}} \frac{\partial \ln g_{c}}{\partial \varphi}
$$

On the other hand, by comparing 7 the $\phi$-dependent terms in (12) to the basic action (11) (both being considered at the cut-off scale $\Lambda_{c}$ ), we see that the coefficients $d_{g}$ and $d_{m_{i}}$ have the effect of adding some $\phi$-dependence in the values of $g_{c}$ and $m_{i}\left(\Lambda_{c}\right)$ of the form

$$
\frac{\partial \ln g_{c}}{\partial \varphi}=-d_{g} \frac{\beta_{c}}{g_{c}}, \quad \frac{\partial \ln m_{i}\left(\Lambda_{c}\right)}{\partial \varphi}=d_{m_{i}}+\gamma_{c} d_{g} .
$$

Inserting these results in the $\varphi$-derivatives of $\Lambda_{3}$ and $m_{i}\left(\Lambda_{3}\right)$ derived above, finally leads (thanks to the cancellation of the $\gamma_{c}$-dependent contribution in the derivative of the masses) to the simple results

$$
\frac{\partial \ln \Lambda_{3}}{\partial \varphi}=d_{g}, \quad \frac{\partial \ln m_{i}\left(\Lambda_{3}\right)}{\partial \varphi}=d_{m_{i}} .
$$

\footnotetext{
${ }^{7}$ In doing this comparison it is useful, as explained above for the Maxwell action, to provisionally use a 'geometric' normalization of the gluon field, i.e. to absorb $g_{3}$ in $A^{A}$.
} 
Summarizing: the physical meaning of the five dilaton-coupling coefficients $d_{a}=d_{e}, d_{g}, d_{m_{e}}, d_{m_{u}}, d_{m_{d}}$ is (at the linear level in $\phi$ ) to introduce a $\phi$-dependence in the parameters entering the low-energy physics of the form

$$
\begin{aligned}
\Lambda_{3}(\varphi) & =\left(1+d_{g} \varphi\right) \Lambda_{3} \\
\alpha(\varphi) & =\left(1+d_{e} \varphi\right) \alpha \\
m_{e}(\varphi) & =\left(1+d_{m_{e}} \varphi\right) m_{e} \\
{\left[m_{i}\left(\Lambda_{3}\right)\right](\varphi) } & =\left(1+d_{m_{i}} \varphi\right) m_{i}\left(\Lambda_{3}\right), i=u, d .
\end{aligned}
$$

\subsection{Ratios of dimensional parameters}

Note that a consequence of these equations is that the dimensionless ratios $m_{e} / \Lambda_{3}, m_{u}\left(\Lambda_{3}\right) / \Lambda_{3}, m_{d}\left(\Lambda_{3}\right) / \Lambda_{3}$ depend on $\varphi$ through the ratios $\left(1+d_{m_{i}} \varphi\right) /\left(1+d_{g} \varphi\right) \simeq\left(1+\left(d_{m_{i}}-d_{g}\right) \varphi\right)$. In other words, the $\varphi$ sensitivity of these dimensionless ratios is

$$
\frac{\partial \ln \left[m_{i}\left(\Lambda_{3}\right) / \Lambda_{3}\right]}{\partial \varphi}=d_{m_{i}}-d_{g} .
$$

Note that this involves only the differences $d_{m_{i}}-d_{g}$. In particular, when the mass couplings $d_{m_{i}}$ are taken to be all equal to $d_{g}$, the effect of the $\phi$ couplings is equivalent to introducing a $\phi$ dependence only in $\Lambda_{3}$ and $\alpha$. This fact can also be seen by means of the formulation (18) of the $d_{g}$ coupling. Indeed, when $d_{m_{i}}=d_{g}$ the sum of (18) and of the massterm couplings is equivalent to having a coupling between $\phi$ and the sum of the anomalous, $T_{g}^{\mathrm{anom}}$, and of the non-anomalous, $T_{g}^{\text {non anom }}$, parts of the trace of the total stress-energy tensor. Therefore, modulo electromagnetic effects, this would imply that $\phi$ couples to the trace of the total stress-energy tensor, i.e. (using the virial theorem) that $\phi$ couples to the total mass of the hadron. In this particular case, the only violations of the EP would come from electromagnetic effects.

However, in view of the fact that the physics which determines (in the Standard Model) the masses of the leptons and quarks involves the symmetry breaking of the electroweak sector, and, in particular, the VEV of the Higgs field, it does not seem a priori likely that a fundamental theory describing the high-energy couplings of the dilaton can ensure such a universal feature. From this point of view, one can consider our final results (26) as useful general parametrizations of the low-energy dilaton couplings, independently of the complicated physics that might connect these parameters to an eventual high-energy description of the $\phi$ couplings to the fields entering the basic Lagrangian. For example, heavy quarks do not enter the 
field couplings (12), but they enter in the relation between the QCD scale $\Lambda_{3}$ (describing the physics at scales $\lesssim 1 \mathrm{GeV}$ ) and the high-energy boundary conditions, $\Lambda_{c}, g_{c}$. Therefore, the parametrization of $d_{g}$ in (26) implicitly takes into account the effect of heavy quarks. [Ref. [15] showed how to explicitly take into account the effect of heavy quarks, and it is easily checked that their results are in agreement with the first equation in (26).]

We can use the above results to rewrite the expression of the scalar couplings to matter (3), (77) in a useful form. As the Planck scale $1 / \kappa$ does not directly enter physics at the QCD scale (besides its possible impact on determining $\Lambda_{3}$ via Eq.(20) ), we can always write the mass of an atom as

$$
m_{A}=\Lambda_{3} M_{A}\left(\frac{m_{u}}{\Lambda_{3}}, \frac{m_{d}}{\Lambda_{3}}, \frac{m_{e}}{\Lambda_{3}}, \alpha\right)
$$

where $M_{A}$ is a dimensionless quantity, which is a function of the four indicated dimensionless quantities, say (for later convenience)

$$
\left(k_{u}, k_{d}, k_{e}, k_{\alpha}\right) \equiv\left(\frac{m_{u}}{\Lambda_{3}}, \frac{m_{d}}{\Lambda_{3}}, \frac{m_{e}}{\Lambda_{3}}, \alpha\right) .
$$

Using this notation, the scalar coupling to matter Eq.(7) can be rewritten (when working in the Einstein frame) as

$$
\alpha_{A}=d_{g}+\bar{\alpha}_{A}
$$

where $d_{g}=\frac{\partial \ln \Lambda_{3}}{\partial \varphi}$ is a universal (non EP-violating) contribution to $\alpha_{A}$, and where the EP-violating part $\bar{\alpha}_{A}$ is given by

$$
\bar{\alpha}_{A} \equiv \frac{\partial \ln M_{A}}{\partial \varphi}=\frac{1}{M_{A}} \frac{\partial M_{A}}{\partial \varphi}=\frac{1}{M_{A}} \sum_{a=u, d, e, \alpha} \frac{\partial M_{A}}{\partial \ln k_{a}} \frac{\partial \ln k_{a}}{\partial \varphi} .
$$

The logarithmic derivatives of the $k_{a}$ are given by Eq. (26), so that we can write more explicitly $\bar{\alpha}_{A}$ as the following sum of four contributions:

$$
\bar{\alpha}_{A}=\frac{1}{M_{A}} \frac{\partial M_{A}}{\partial \varphi}=\frac{1}{M_{A}}\left[\sum_{a=u, d, e}\left(d_{m_{a}}-d_{g}\right) \frac{\partial M_{A}}{\partial \ln k_{a}}+d_{e} \frac{\partial M_{A}}{\partial \ln \alpha}\right] .
$$

\subsection{Redefining the quark mass parameters}

In the following, we will find it convenient to work with the symmetric and antisymmetric combinations of the light quark masses, namely

$$
\hat{m}=\frac{1}{2}\left(m_{d}+m_{u}\right), \quad \delta m=\left(m_{d}-m_{u}\right)
$$


Working in terms of $\hat{m}$ and $\delta m$, means working in terms of mass terms of the form

$$
m_{d} \bar{d} d+m_{u} \bar{u} u=\hat{m}(\bar{d} d+\bar{u} u)+\frac{1}{2} \delta m(\bar{d} d-\bar{u} u)
$$

which couple to the dilaton as

$$
\mathcal{L}_{\phi}=\ldots-\kappa \phi\left[d_{\hat{m}} \hat{m}(\bar{d} d+\bar{u} u)+\frac{d_{\delta m}}{2} \delta m(\bar{d} d-\bar{u} u)\right]
$$

These definitions are such that, for instance, the coupling of $\varphi$ to $\hat{m}$ is equivalent to a Hamiltonian coupling of the form,

$$
\mathcal{H}=\ldots .+\left(1+d_{\hat{m}} \varphi\right) \hat{m}(\bar{u} u+\bar{d} d),
$$

i.e. to introducing a $\varphi$ dependence in the average light quark mass of the type $\hat{m}(\varphi)=\left(1+d_{\hat{m}} \varphi\right) \hat{m}$.

The link between these new dilaton-coupling coefficients and the previous ones reads

$$
d_{\hat{m}} \equiv \frac{\partial \ln \hat{m}}{\partial \varphi}=\frac{d_{m_{d}} m_{d}+d_{m_{u}} m_{u}}{m_{d}+m_{u}}, d_{\delta m} \equiv \frac{\partial \ln \delta m}{\partial \varphi}=\frac{d_{m_{d}} m_{d}-d_{m_{u}} m_{u}}{m_{d}-m_{u}} .
$$

In term of this notation (32) reads

$$
\begin{aligned}
\bar{\alpha}_{A}= & \frac{1}{M_{A}}\left[\left(d_{\hat{m}}-d_{g}\right) \hat{m} \frac{\partial M_{A}}{\partial \hat{m}}+\left(d_{\delta m}-d_{g}\right) \delta m \frac{\partial M_{A}}{\partial \delta m}\right. \\
& \left.+\left(d_{m_{e}}-d_{g}\right) m_{e} \frac{\partial M_{A}}{\partial_{m_{e}}}+d_{e} \alpha \frac{\partial M_{A}}{\partial \alpha}\right] .
\end{aligned}
$$

As displayed in Eq. (38), $\bar{\alpha}_{A}$ is naturally decomposed into a sum of four contributions, which are linear in the four dilaton couplings: $d_{m_{a}}-d_{g}$, or $d_{e}$. Another linear decomposition can also be applied to the various terms in $\bar{\alpha}_{A}$ : namely the one corresponding to the various terms in Eq. (9). Regrouping some terms in these two possible linear decompositions, we shall find convenient in our calculations (before coming back to the more theoretically rooted decomposition (38) ) to decompose $\bar{\alpha}_{A}$ into three contributions:

$$
\bar{\alpha}_{A}=\bar{\alpha}_{A}^{\mathrm{rmwo} \cdot \mathrm{EM}}+\bar{\alpha}_{A}^{\text {bind }}+\bar{\alpha}_{A}^{d_{e}}
$$

where $\bar{\alpha}_{A}^{\text {rm wo. EM }}$ denotes the contribution coming from the terms linear in the quark and electron masses in the rest-mass contribution (10) to $m_{A}$ (without the electromagnetic contributions), where $\bar{\alpha}_{A}^{\text {bind }}$ denotes the contribution coming from the nuclear binding energy $E^{\text {bind }}$ in Eq. (9), i.e.

$$
\bar{\alpha}_{A}^{\text {bind }}=\frac{1}{M_{A}} \frac{\partial\left(E^{\text {bind }}(\varphi) / \Lambda_{3}\right)}{\partial \varphi}(\text { with fixed } \alpha),
$$


and where $\bar{\alpha}_{A}^{d_{e}}$ denotes the total electromagnetic contribution, coming both from the EM contributions to the masses of the nucleons, and from the nuclear Coulomb energy term $E_{1}$, which is a part of $E^{\text {bind }}$ in Eq. (9). Note that $\bar{\alpha}_{A}^{d_{e}}$ collects the terms in $\bar{\alpha}_{A}$ which are proportional to the EM dilaton coupling $d_{e}$, i.e. which come from the $\varphi$ sensitivity of the fine-structure constant $\alpha$. This is why we have added in the definition of $\bar{\alpha}_{A}^{\text {bind }}$ above the fact that one must keep $\alpha$ constant when computing it. As we shall see, the Coulomb energy term plays a special role in that it depends both on nuclear-binding effects, and on EM ones. As a consequence it will give two separate contributions: one to $\bar{\alpha}_{A}^{\text {bind }}$ and one to $\bar{\alpha}_{A}^{d_{e}}$.

\section{Analysis of scalar couplings to the binding en- ergy of nuclei}

We will first focus on the scalar coupling to the nuclear binding energy, Eq. (40), because this term has not yet received a satisfactory treatment in the literature.

When dealing with nuclear binding it is convenient to work with the (half) sum and difference of the light quark 8 masses, $\hat{m}$ and $\delta m$, as introduced above. Indeed, the quark-mass dependence of nuclear binding is dominated by its dependence on the average light quark mass $\hat{m}$ because pion exchanges yield the dominant contribution to nuclear binding, and pion masses are proportional to $\hat{m}$, while they are insensitive to the difference in quark masses. [ The quark mass difference $\delta m$ is important for the neutron and proton masses, and will enter the computation below of the rest-mass contribution to EP violation.]

As explained above, the $\varphi$ dependence of $d_{\hat{m}}$ implies the following result for the 'nuclear binding energy' contribution, Eq. (40), to EP violation:

$$
\bar{\alpha}_{A}^{\text {bind }}=\frac{\left(d_{\hat{m}}-d_{g}\right)}{m_{A}} \hat{m} \frac{\partial E^{\text {bind }}}{\partial \hat{m}}
$$

In QCD, because the pion is almost a Goldstone boson of the dynamically broken chiral symmetry, the pion mass-squared is linear in the quark mass, $m_{\pi}^{2} \simeq b_{0} \Lambda_{3} \hat{m}$, where $b_{0}$ is a pure number. This relation is accurate in the

\footnotetext{
${ }^{8}$ As explained above, heavy quarks are assumed to have been integrated out from the theory, thereby producing a shift in the QCD scale $\Lambda_{3}$, and its associated dilaton coupling $d_{g}$. The effect of the strange quark, which is intermediate between heavy and light, is discussed in the appendix.
} 
physical region, so that we can translate our formula into one involving the pion mass,

$$
\bar{\alpha}_{A}^{\text {bind }}=\frac{\left(d_{\hat{m}}-d_{g}\right)}{m_{A}} m_{\pi}^{2} \frac{\partial E^{\text {bind }}}{\partial m_{\pi}^{2}}
$$

Our major task then translates into knowing the dependence of nuclear binding on the mass of the pion.

The semi-empirical mass formula describes the binding energy $m_{A}-$ $m_{A}^{\text {rest mass }}$ through the following terms:

$$
m_{A}-m_{A}^{\text {rest mass }}=E^{\text {bind }},
$$

where the nuclear binding energy is approximately described as

$$
E^{\text {bind }}=-a_{v} A+a_{s} A^{2 / 3}+a_{a} \frac{(A-2 Z)^{2}}{A}+a_{c} \frac{Z(Z-1)}{A^{1 / 3}}-\delta \frac{a_{p}}{A^{1 / 2}} .
$$

The various contributions to the nuclear binding energy 9 are called, respectively the volume energy, the surface energy the asymmetry energy, the Coulomb energy and the pairing energy. [In the latter, $\delta=\frac{1}{2}\left[(-)^{N}+(-)^{Z}\right]$, i.e. $\delta=+1$ for even-even nuclei, $\delta=-1$ for odd-odd nuclei and $\delta=0$ otherwise.] Typical fit values for these parameters are [23] $a_{v}=16 \mathrm{MeV}, a_{s}=$ $17 \mathrm{MeV}, a_{a}=23 \mathrm{MeV}, a_{p}=12 \mathrm{MeV}, a_{c}=0.717 \mathrm{MeV}$. Note that, here and in the following, the unit of $1 \mathrm{MeV}$ is supposed to represent a fixed fraction of the QCD mass scale, say $\simeq 10^{-3} \Lambda_{3}$ if we use, as indicated above, a reference value $g_{*}$ for $g_{3}$ such that $\Lambda_{3} \simeq 1 \mathrm{GeV}$.]

The $\hat{m}$ sensitivity of $E^{\text {bind }}$ comes from the $\hat{m}$ sensitivity of the various coefficients $a_{v}, a_{s}, a_{a}, a_{c}, a_{p}$ (taken in units of $\Lambda_{3}$ ). We shall discuss successively the $\hat{m}$ sensitivities of: (i) $a_{v}$ and $a_{s}$, (ii) $a_{a}$, and (iii) $a_{c}$. Concerning the pairing interaction term $a_{p}$ we found that it was subdominant in our final results because it is down by a factor of $A^{7 / 6}$ compared to our primary $A$ dependence. Even when allowing for variations with quark mass comparable to that of the asymmetry energy we found that it is negligible in the end, so we drop it at this stage.

\subsection{The central nuclear force terms: $a_{v}$ and $a_{s}$}

Let us first consider the terms proportional to $a_{v}$ and $a_{s}$. They come from the isospin symmetric central nuclear force, which is the dominant contribution

\footnotetext{
${ }^{9}$ Please be aware of a dual notation in that the letter $A$ is used both as a label for a certain type of atom, and, in the semi-empirical mass formula, as a notation for the mass number $A=Z+N$.
} 
in the binding of heavy nuclei. Our previous work [21, 22] shows that this component has an enhanced dependence on the quark masses and hence it has an enhanced coupling to a dilaton. This large dependence comes because the central potential involves competing effects of an intermediate range attractive force and a shorter range repulsive force. The cancelation between these two effects (which are individually of order $\pm 100 \mathrm{MeV}$ per nucleon) lead to a binding energy which is quite small on the QCD scale (namely of order $-10 \mathrm{MeV}$ per nucleon). However, the attractive force is far more sensitive to pion masses because it involves two pion exchange. Changing the pion mass a modest amount upsets the cancelation of the two components and leads to a larger effect than might naively be expected.

The central force is parameterized by two terms denoting the volume energy and the surface energy,

$$
E^{\text {bind }}=-a_{v} A+a_{s} A^{2 / 3}+\text { residual terms }
$$

The central potential is isospin symmetric, and can involve exchanges which carry angular momentum quantum numbers 0 or 1 . The work of Ref. [24] uses a general basis of contact interactions [25] to quantify these contributions to nuclear binding. This parameterization only assumes that the interactions have a range which is smaller than the momentum in nuclei $k \sim 200 \mathrm{MeV}$. The dominant contact interactions are found to be those of an attractive scalar and a repulsive vector, describing the integrated effects of the potentials. They are parameterized by strengths $G_{S}\left(G_{V}\right)$ for the scalar (vector) channel. We can then use the results of Ref. [24] to give the binding energy as a function of these strengths, normalized to their physical values, by defining

$$
\eta_{S} \equiv \frac{G_{S}}{\left.G_{S}\right|_{\text {physical }}}, \quad \eta_{V} \equiv \frac{G_{V}}{\left.G_{V}\right|_{\text {physical }}}
$$

This results in

$$
E^{\text {bind }}=-\left(120 A-97 A^{2 / 3}\right) \eta_{S}+\left(67 A-57 A^{2 / 3}\right) \eta_{V}+\text { residual terms }
$$

where the numbers are in units of $\mathrm{MeV}$. One can see here the cancelation between the primary terms as each is larger than their sum. Of these two contributions, our calculations indicate that it is the scalar channel $\left(\eta_{S}\right)$ that has the most important effect. This is because the scalar channel is dominated by the exchange of two pions, which is highly sensitive to the pion mass. While the two pion contribution is often parameterized by an effective sigma meson, the low energy exchange of two pions is required in 
chiral perturbation theory and is calculable10. This accounts for much of the strength typically ascribed to the sigma [26]. The vector interaction has a very small low energy contribution from three pions, and estimates of the quark mass dependence of the mass and couplings of a massive vector boson indicate a tiny residual contribution [21].

With these results we have argued that the main contribution is the variation of the scalar strength with quark mass,

$$
\bar{\alpha}_{A}^{\text {bind }}=-\frac{\left(d_{\hat{m}}-d_{g}\right)}{m_{A}}\left(120 A-97 A^{2 / 3}\right) m_{\pi}^{2} \frac{\partial \eta_{S}}{\partial m_{\pi}^{2}}
$$

We use the result of Ref. [21, displayed in Fig 1 showing the scalar strength as a function of the pion mass. This variation arose almost entirely from the threshold modification in the two pion effects at low energy, where the chiral techniques are most reliable and where we expect the greatest sensitivity to a change in the mass $[28,29]$. We can use this directly to obtain

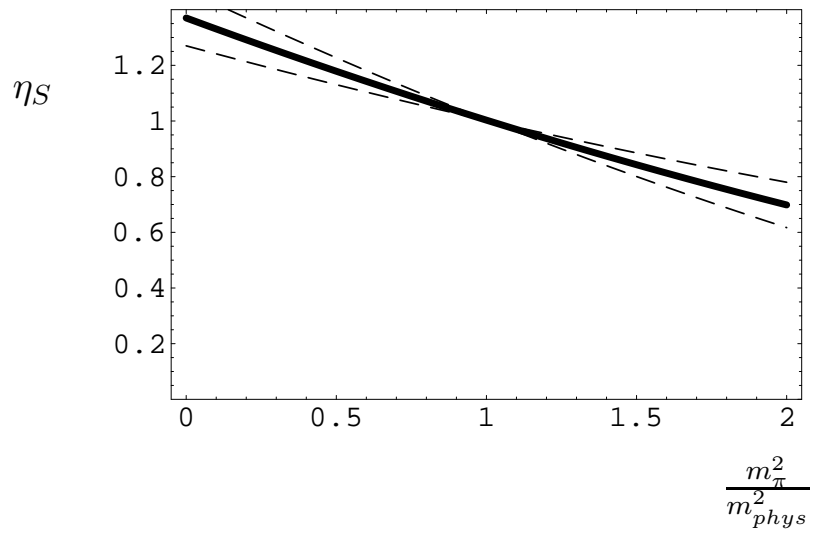

Figure 1: The value of the scalar strength $\eta_{S}$ as a function of the pion mass.

$$
\hat{m} \frac{\partial \eta_{S}}{\partial \hat{m}}=m_{\pi}^{2} \frac{\partial \eta_{S}}{\partial m_{\pi}^{2}}=-0.35 \pm 0.10
$$

The error bar comes from uncertainties in the chiral expansion. We will not display the error bar in subsequent formulas, but all results in the binding energy carry this level of uncertainty. Our final result for the central

\footnotetext{
${ }^{10}$ Other estimates of mass dependence [27] have not explicitly taken into account this low energy effect.
} 
dependence in the dilaton coupling is

$$
\begin{aligned}
\left.\bar{\alpha}_{A}^{\text {bind }}\right|_{\text {central }} & =\frac{\left(d_{\hat{m}}-d_{g}\right)}{m_{A}}\left(42 A-34 A^{2 / 3}\right)(\mathrm{MeV}) \\
& \approx\left(d_{\hat{m}}-d_{g}\right) F_{A}\left(0.045-\frac{0.036}{A^{1 / 3}}\right) .
\end{aligned}
$$

In the final line we have introduced the notation

$$
F_{A} \equiv \frac{A m_{\mathrm{amu}}}{m_{A}}
$$

where $m_{\mathrm{amu}}=931 \mathrm{MeV}$ is the atomic mass unit (i.e. the nucleon mass $m_{N}=$ $939 \mathrm{MeV}$ minus the average binding energy per nucleon, $\simeq 8 \mathrm{MeV}$ ). The factor $F=A m_{\mathrm{amu}} / m_{A}$ remains quite close to one all over the periodic table (modulo $O\left(10^{-3}\right)$ ). Note that our result Eq. (50) for the light-quark-mass $(\hat{m})$ dependence is significantly larger (by a factor 2.2) than the estimate used by Dent [17]. Indeed, Eq. (50) corresponds, say for the crucial surface energy, to a logarithmic sensitivity $\partial \ln a_{s} / \partial \ln \hat{m}=-34 \mathrm{MeV} / a_{s}=-2$, while Ref. [17] estimated $\partial \ln a_{s} / \partial \ln \hat{m} \simeq-0.9$.

\subsection{The asymmetry energy term: $a_{a}$}

Let us now discuss the $\varphi$ sensitivity of the asymmetry energy $\propto a_{a}$ which is, after the volume, surface and Coulomb terms, the fourth dominant contribution to $E^{\text {bind }}$. The asymmetry energy has two components. The first comes from the Pauli principle which requires that, when there is an excess of neutrons over protons, the extra neutrons must be placed into higher energy states than the protons. The other component is due to the nuclear force in which the isospin dependent interactions create a stronger attraction for an neutron and proton compared to two neutrons or two protons.

The asymmetry energy has been calculated by Serot and Walecka 30 in the same framework that we use in our work on nuclear matter [22]. This takes the form

$$
a_{a}=\frac{k_{F}^{2}}{6 \sqrt{M_{*}^{2}+k_{F}^{2}}}+\frac{G_{\rho}}{12 \pi^{2}} k_{F}^{3}
$$

where

$$
M_{*}=m_{N}\left(1+\frac{\gamma G_{S} k_{F}^{3}}{6 \pi^{2}}\right)
$$

is the nucleon mass modified by interactions in nuclear matter (with $G_{S}<0$ so that $\left.M_{*}<m_{N}\right)$. For isoscalar nuclear matter we have $\gamma=4$. In meson 
exchange models $G_{\rho}=g_{\rho}^{2} / m_{\rho}^{2}$ and $G_{S}=-g_{\sigma}^{2} / m_{\sigma}^{2}$ are the vector meson and scalar coupling strengths. The $k_{F}^{3}$ dependence in the second term in $a_{a}$ comes from a calculation of the nuclear density in terms of the Fermi momentum $k_{F}$.

As mentioned above, our estimates indicate that the mass dependence of the vector meson coupling strength is weak. However, the Fermi momentum depends on the scalar strength, which has a sizeable mass variation. The Fermi momentum increases as the scalar strength increases. We calculate this through our work on nuclear matter in which we solve for the Fermi momentum as a function of the scalar strength (e.g. see Fig. 4 of [22]). More precisely, using our approximate analytical model, Eq. (17) of [22], with the values $G_{S}=-355.388 \mathrm{GeV}^{-2}$, and $G_{S}=+262.89 \mathrm{GeV}^{-2}$ (which entail the phenomenologically good values $a_{v}=15.75 \mathrm{MeV}$ and $k_{F}=1.30 \mathrm{fm}^{-1}$ ) we find that

$$
\frac{\partial \ln k_{F}}{\partial \ln G_{S}} \simeq 0.525
$$

Using that dependence we find that both components of the asymmetry energy in Eq. (52) vary in the same direction with the scalar strength. The kinetic contribution (first term) varies with a logarithmic rate $\simeq 2.54$, while the other one varies like $k_{F}^{3}$, i.e. with a logarithmic rate $3 \times 0.525=1.575$. The combination of the two contributions then varies with a rate

$$
\frac{\partial \ln a_{a}}{\partial \ln G_{S}} \simeq 2.35
$$

Combining this variation with the logarithmic mass variation Eq. (49) of the scalar strength $G_{S}$ then yields

$$
m_{\pi}^{2} \frac{\partial a_{a}}{\partial m_{\pi}^{2}}=\frac{\partial a_{a}}{\partial G_{S}} m_{\pi}^{2} \frac{\partial G_{S}}{\partial m_{\pi}^{2}}=-0.82 a_{a}=-19 \mathrm{MeV}
$$

Note that our framework shows that $\partial \ln a_{a} / \partial \ln \hat{m} \simeq-0.82$ is rather different from $\partial \ln a_{s} / \partial \ln \hat{m}=-2$. This shows again the subtlety of quark-mass effects in nuclear physics.

\subsection{The Coulomb energy term: $a_{c}$}

The Coulomb energy also has a dependence on the strong interaction coupling terms. Dimensionally this is because the electromagnetic coupling $\alpha$ is dimensionless, so that the overall energy scale associated with $a_{c}$ comes from the nuclear interactions. Physically, this dependence is also logical because the Coulomb energy depends on how tightly the nucleons are packed together. We estimate this effect in this subsection. 
An approximate analytic expression for the coefficient of the Coulomb contribution to the nuclear binding energy is $a_{c} \simeq(3 / 5) \alpha / r_{0}$ where $r_{0} \simeq$ $1.2 \mathrm{fm}$ is the scaled nuclear radius: $r_{A}=r_{0} A^{1 / 3}$. Writing that the total baryonic number within the volume of the nucleus, i.e. $\rho_{B} 4 \pi r_{A}^{3} / 3$ (with $\rho_{B}=$ $\left.\gamma k_{F}^{3} /\left(6 \pi^{2}\right)\right)$ is equal to $A$, one gets the link $k_{F} r_{0}=(9 \pi / 8)^{1 / 3}$. Therefore, $r_{0}$ varies inversely proportionally to $k_{F}$, so that the above result shows that $a_{c} \propto \alpha k_{F}$. This yields a logarithmic sensitivity of $a_{c}$ to variations of $G_{S}$ with the same rate as $k_{F}$ itself, i.e. 0.525 , as quoted above. Multiplying this rate by the rate -0.35 of Eq. (49), then yields

$$
\hat{m} \frac{\partial E_{1}}{\partial \hat{m}}=-0.184 a_{c} \frac{Z(Z-1)}{A^{1 / 3}}=-0.13 \frac{Z(Z-1)}{A^{1 / 3}} \mathrm{MeV}
$$

\subsection{The complete scalar coupling to the binding energy}

Combining our partial results, we finally obtain for $\bar{\alpha}_{A}^{\text {bind }}$ the following sum

$$
\begin{aligned}
\bar{\alpha}_{A}^{\text {bind }} & =\left(d_{\hat{m}}-d_{g}\right) F_{A} \\
& \times\left[0.045-\frac{0.036}{A^{1 / 3}}-0.020 \frac{(A-2 Z)^{2}}{A^{2}}-1.42 \times 10^{-4} \frac{Z(Z-1)}{A^{4 / 3}}\right]
\end{aligned}
$$

In writing this result, we have, as above, factorized $F_{A}=A m_{\mathrm{amu}} / m_{A}$.

\section{Scalar couplings to the rest mass of atoms}

In this section we study the first term on the r.h.s. of Eq. (39), i.e. the contribution to $\bar{\alpha}_{A}$ coming from the $\varphi$ sensitivity of the rest masses of the low-energy constituents of atoms, namely protons, neutrons and electrons (à la [12]).

In view of the expression Eq. (34) for the mass terms of the light quarks, we can write the masses of the nucleons as 31]

$$
\begin{aligned}
& m_{p}=m_{N 3}+\sigma-\frac{1}{2} \delta+C_{p} \alpha, \\
& m_{n}=m_{N 3}+\sigma+\frac{1}{2} \delta+C_{n} \alpha
\end{aligned}
$$

where $m_{N 3}$ is the nucleon mass in the "chiral limit" of massless light11 quarks, and where the electromagnetic contributions $C_{p} \alpha, C_{n} \alpha$ will be ignored here and treated in the next section. The quantities $\sigma$ and $\delta$ in

\footnotetext{
${ }^{11}$ In the present treatment, we absorb in $m_{N 3} \propto \Lambda_{3}$ the EP non violating effect of the strange quark; see the appendix.
} 
Eq. (59) denote the matrix elements of the isoscalar $(\propto \bar{d} d+\bar{u} u)$ and isovector $(\propto \bar{d} d-\bar{u} u)$ terms in a neutron state:

$$
\begin{aligned}
\sigma & =\langle n|\hat{m}(\bar{d} d+\bar{u} u)| n\rangle \\
\delta & =\left\langle n\left|\left(m_{d}-m_{u}\right)(\bar{d} d-\bar{u} u)\right| n\right\rangle
\end{aligned}
$$

These combinations of the quark mass contributions to the individual nucleons are reasonably well known. The isoscalar contribution is related to the $\pi N$ sigma term and has the value $\sigma=45 \mathrm{MeV}$ [33]. The isovector difference can be obtained by $\mathrm{SU}(3)$ sum rules

$$
\delta=\frac{m_{d}-m_{u}}{m_{s}-\hat{m}}\left[m_{\Xi}-m_{\sigma}\right]=3.1 \mathrm{MeV}
$$

The $\varphi$ sensitivity of the rest mass contribution (without the EM contribution) of an atom,

$$
m_{A}^{\mathrm{rm} \text { wo. } \mathrm{EM}}=A m_{N_{3}}+A \sigma+\frac{1}{2}(N-Z) \delta+Z m_{e},
$$

comes from the fact that $\sigma \propto \hat{m}(\varphi), \delta \propto \delta m(\varphi)$, and from the $\varphi$ dependence of $m_{e}$. Using our general results above, we therefore have

$$
\begin{aligned}
\bar{\alpha}_{A}^{\mathrm{rm} \text { wo. EM }} & =\left(d_{\hat{m}}-d_{g}\right) \frac{A \sigma}{m_{A}}+\frac{1}{2}\left(d_{\delta_{m}}-d_{g}\right) \frac{(N-Z) \delta}{m_{A}} \\
& +\left(d_{m_{e}}-d_{g}\right) \frac{Z m_{e}}{m_{A}} .
\end{aligned}
$$

Inserting the numerical values of $\sigma, \delta$ and $m_{e}$ yields

$$
\begin{aligned}
\bar{\alpha}_{A}^{\mathrm{rm} \text { wo. EM }} & \simeq F_{A}\left[0.048\left(d_{\hat{m}}-d_{g}\right)+0.0017\left(d_{\delta_{m}}-d_{g}\right) \frac{A-2 Z}{A}\right. \\
& \left.+5.5 \times 10^{-4}\left(d_{m_{e}}-d_{g}\right) \frac{Z}{A}\right] .
\end{aligned}
$$

\section{$6 \quad$ Electromagnetic effects}

In this section, we review the electromagnetic coupling, which is contained in the Lagrangian, i.e. the contribution

$$
\alpha_{A}^{\left(d_{e}\right)}=\frac{d_{e}}{m_{A}} \alpha \frac{\partial m_{A}}{\partial \alpha}
$$


The main electromagnetic effects in the atomic masses come from the electromagnetic shifts in the nucleon masses and from the electromagnetic contribution to nuclear binding, $E_{1}$.

$$
\alpha_{A}^{\left(d_{e}\right)}=\frac{d_{e}}{m_{A}}\left[Z \alpha \frac{\partial m_{p}}{\partial \alpha}+(A-Z) \alpha \frac{\partial m_{n}}{\partial \alpha}+\alpha \frac{\partial E_{1}}{\partial \alpha}\right]
$$

We follow Gasser and Leutwyler [31] in the estimate of the electromagnetic portions of the proton and neutron masses

$$
\alpha \frac{\partial m_{p}}{\partial \alpha}=C_{p}=0.63 \mathrm{MeV} \quad \alpha \frac{\partial m_{n}}{\partial \alpha}=C_{n}==-0.13 \mathrm{MeV}
$$

The electromagnetic binding is known from the semi-empirical mass formula

$$
\alpha \frac{\partial E_{1}}{\partial \alpha}=a_{c} \frac{Z(Z-1)}{A^{1 / 3}}
$$

with $a_{c}=0.717 \mathrm{MeV}$. These combine to yield

$$
\bar{\alpha}_{A}^{\left(d_{e}\right)}=d_{e} F_{A}\left[-1.4+8.2 \frac{Z}{A}+7.7 \frac{Z(Z-1)}{A^{4 / 3}}\right] \times 10^{-4}
$$

As above, the factor $F_{A}=A m_{\mathrm{amu}} / m_{A}$ can be replaced by one in lowest approximation.

\section{Implications for the Equivalence Principle}

\subsection{General parameterization}

Summarizing our results, the dilaton coupling to an atom can be written as

$$
\alpha_{A}=d_{g}+\bar{\alpha}_{A}^{\text {rm wo. EM }}+\bar{\alpha}_{A}^{\text {bind }}+\bar{\alpha}_{A}^{\left(d_{e}\right)}
$$

where $\bar{\alpha}_{A}^{\text {rm wo. EM }}$ is given by Eq. (64), $\bar{\alpha}_{A}^{\text {bind }}$ by Eq. (58), and $\bar{\alpha}_{A}^{\left(d_{e}\right)}$ by Eq. (69). It will be convenient for the following to rewrite this result as

$$
\alpha_{A}=d_{g}+\bar{\alpha}_{A}
$$

with the decomposition

$$
\bar{\alpha}_{A}=\left[\left(d_{\hat{m}}-d_{g}\right) Q_{\hat{m}}+\left(d_{\delta m}-d_{g}\right) Q_{\delta m}+\left(d_{m_{e}}-d_{g}\right) Q_{m_{e}}+d_{e} Q_{e}\right]_{A}
$$


where $Q_{k_{a}}$ can be thought of as the ' dilaton charge' coupled to the parameter $k_{a}$. These are given by

$$
\begin{gathered}
Q_{\hat{m}}=F_{A}\left[0.093-\frac{0.036}{A^{1 / 3}}-0.020 \frac{(A-2 Z)^{2}}{A^{2}}-1.4 \times 10^{-4} \frac{Z(Z-1)}{\left.A^{4 / 3}\right]}\right. \\
Q_{\delta m}=F_{A}\left[0.0017 \frac{A-2 Z}{A}\right] \\
Q_{m_{e}}=F_{A}\left[5.5 \times 10^{-4} \frac{Z}{A}\right]
\end{gathered}
$$

and

$$
Q_{e}=F_{A}\left[-1.4+8.2 \frac{Z}{A}+7.7 \frac{Z(Z-1)}{A^{4 / 3}}\right] \times 10^{-4} .
$$

Here, as above, the factor $F_{A}$ denotes $F_{A} \equiv A m_{\mathrm{amu}} / m_{A}$ (it can be replaced by one in lowest approximation).

\subsection{Relation to theoretical expectations}

Note that all the various contributions to the non-universal part $\bar{\alpha}_{A}$ of $\alpha_{A}=$ $d_{g}+\bar{\alpha}_{A}$ contain small numerical coefficients in front of the various basic dilaton couplings $d_{g}, d_{e}, d_{\hat{m}}, d_{\delta m}, d_{m_{e}}$. It is therefore a priori probable that the composition-dependent part $\bar{\alpha}_{A}$ is small compared to the compositionindependent 12 part $\alpha_{A}^{\text {c.i. }}=d_{g}$.

We recall that the latter composition-independent part is, in principle, accessible in various experimental tests of relativistic gravity. For instance, in the notation of tests of post-Newtonian gravity, $\alpha_{A}^{\text {c.i. }}=d_{g}$, is related to the Eddington parameter $\gamma$ via (see, e.g., [14])

$$
\gamma-1=-2 \frac{d_{g}^{2}}{1+d_{g}^{2}} \simeq-2 d_{g}^{2}
$$

The most precise current test of relativistic gravity [32] constrain $(\gamma-1) / 2$, i.e. $d_{g}^{2}$ at the level

$$
d_{g}^{2} \simeq \frac{1-\gamma}{2}<10^{-5}
$$

\footnotetext{
${ }^{12}$ Actually, if we define the composition-independent part of $\alpha_{A}$ by some average over the composition of the bodies relevant for the considered gravity tests, $\alpha_{A}^{\text {c.i. }}$ will have, besides $d_{g}$, a contribution coming from $\bar{\alpha}_{A}$, and notably from terms $\sim 0.1\left(d_{\hat{m}}-d_{g}\right)$ coming fron the QCD binding of nucleons, and the nuclear binding of nuclei. To simplify our discussion we shall assume that these terms are small. It is enough to replace some of our factors $d_{g}$ below by $\alpha_{A}^{\text {c.i. }}=d_{g}^{*} \simeq d_{g}+0.1\left(d_{\hat{m}}-d_{g}\right)+\cdots$ to refine our estimates.
} 
Planned improved solar-system tests might improve this limit to the $10^{-7}$ level. As we are going to see, and as was pointed out by many authors before (see, e.g. [36]), such levels are much less constraining than the ones accessible by experimental tests of the EP.

By contrast to the composition-independent tests whose signals are proportional to $d_{g}^{2}$, the EP-violation signals will all be (see Eq. (8) ) proportional to

$$
\alpha_{E}\left(\alpha_{A}-\alpha_{B}\right) \simeq \alpha^{\text {c.i. }}\left(\bar{\alpha}_{A}-\bar{\alpha}_{B}\right)
$$

Therefore EP signals will involve the product of $d_{g}$ (or rather $d_{g}^{*}=\alpha^{\text {c.i. }}$ ) by one of the other dilaton couplings entering the $\bar{\alpha}_{A}$ 's, i.e. they will be proportional to a combination of terms involving the following four coefficients

$$
d_{g}^{*}\left(d_{\hat{m}}-d_{g}\right), d_{g}^{*}\left(d_{\delta m}-d_{g}\right), d_{g}^{*}\left(d_{m_{e}}-d_{g}\right) \quad \text { or } \quad d_{g}^{*} d_{e}
$$

This raises several issues of direct phenomenological interest: (i) can, in principle, EP experiments measure all four (a priori independent) parameters (80) ?; (ii) are there theoretical arguments suggesting that, among all the EP signals associated to these parameters, some of them might dominate over the others?

Concerning the first question (which has also been addressed in [17]), let us note that if we approximate the factor $F_{A}=A m_{\mathrm{amu}} / m_{A}$ by one ( and $Z(Z-1)$ by $\left.Z^{2}\right)$, the composition dependence of our general dilaton coupling above will vary, along the periodic table, according to

$$
\bar{\alpha}_{A}=a_{0}+\frac{a_{1}}{A^{1 / 3}}+a_{2} \frac{A-2 Z}{A}+a_{3} \frac{(A-2 Z)^{2}}{A^{2}}+a_{4} \frac{Z^{2}}{A^{4 / 3}}
$$

where the five coefficients $a_{0}, \ldots, a_{4}$ are linear combinations (which are easily read off the results above) of the four dimensionless dilaton couplings $d_{\hat{m}}-$ $d_{g}, d_{\delta m}-d_{g}, d_{m_{e}}-d_{g}, d_{e}$. Here the constant offset $a_{0}$ is not measurable 13 in EP experiments. By contrast, EP experiments can, in principle, measure the coefficients of the four different composition-dependences associated with $a_{1}, a_{2}, a_{3}, a_{4}$. Barring some degeneracies, this means that, in principle, a well-devised set of ideal EP experiments could measure the four theoretical parameters (80) [see, e.g., 3] for discussions of the related optimization of the choice of materials in EP experiments, and [17] for an example of the determination of four theoretical parameters from four independent EP data].

\footnotetext{
${ }^{13}$ At least in our approximation $A m_{\mathrm{amu}} / m_{A} \simeq 1$. If one were to keep the small fractional $\left(\sim 10^{-3}\right)$ variations of the ratio $A m_{\mathrm{amu}} / m_{A}$, one might measure part of the $a_{0}$ coefficient.
} 
However, EP experiments will be more likely to detect signals associated with functions of $A$ and $Z$ that vary significantly over the periodic table. From this point of view, two signals, among the four ones in Eq. (81), are likely to be more prominent: namely the ones associated to the parameters $a_{1}$ and $a_{4}$. Indeed, both $A^{-1 / 3}$ and $Z^{2} A^{-4 / 3}$ vary significantly along the periodic table. By contrast, the quantities $(A-2 Z) / A$ and $((A-2 Z) / A)^{2}$ vary only mildly. Indeed, the 'valley' of stable nuclei is located along a specific line in the $A, Z$ plane which is rather close to the $A=2 Z$ (i.e. $N=Z$ ) straight line. Actually, in absence of the Coulomb repulsion between protons, the Pauli principle would favour an equal number of protons and neutrons (cf. the discussion of the asymmetry energy above). The Coulomb effects modify this in favouring a relatively small excess of neutrons over protons. More precisely, the bottom of the valley of stable nuclei is around 23 .

$$
Z_{\text {stable }} \simeq \frac{1}{2} \frac{A}{1+0.015 A^{2 / 3}}
$$

Using this result we see that $(2 Z-A) / A \simeq\left(1+0.015 A^{2 / 3}\right)^{-1}-1$, which is small and whose variation with $A$ is reduced by the small coefficient 0.015 .

In conclusion, the two EP signals that are probably most easily measurable in Eq. (81) are the ones associated to $A^{-1 / 3}$ and $Z^{2} A^{-4 / 3}$. In previous work on the phenomenological consequences of dilaton couplings [3, 12 it was suggested that the EP signal would be essentially proportional to $Z^{2} A^{-4 / 3}$, i.e. related to the Coulomb energy term $\propto d_{e}$ in the results above. Our analysis of the quark-mass sensitivity of nuclear binding is now modifying this conclusion in suggesting that the $\varphi$ dependence of atomic masses will contain, in addition to this Coulomb-related term, another term (related to the quark-mass dependence of nuclear binding), with a $A^{-1 / 3}$ variation over the periodic table.

An important issue is to know whether theoretical considerations can tell us a priori something about the relative order of magnitude of these Coulomb and nuclear terms. In order to discuss this we need to know something about the expected relative magnitude of $d_{g}^{*} d_{e}$ versus $d_{g}^{*}\left(d_{g}-d_{\hat{m}}\right)$, i.e. the relative magnitude of $d_{e}$ versus $d_{g}-d_{\hat{m}}$. We shall next argue that it is theoretically plausible either that $d_{e} \sim d_{g}-d_{\hat{m}}$, or that $d_{e} \sim\left(d_{g}-d_{\hat{m}}\right) / 40$.

Indeed, we have seen above that our dilaton coefficients $d_{g}, d_{\hat{m}}, d_{e}$ where respectively defined as being the logarithmic derivatives of $\Lambda_{3}, \hat{m}, \alpha$. On the other hand, it is natural to consider (at least in string theory) that a dilaton couples with roughly equal strengths to the various terms in the Wilsonian action considered at some high-energy 'cut-off' scale $\Lambda_{c}$, near the string 
scale, i.e. probably near the Planck scale $m_{P}=1 / \kappa \sim 3.44 \times 10^{18} \mathrm{GeV}$. If this is the case, the relative magnitudes of the low-energy dilaton couplings $d_{g}, d_{\hat{m}}, d_{e}$ is determined by the functional dependences that relate the lowenergy quantities $\Lambda_{3}, \hat{m}, \alpha$ to basic couplings at the string, or Planck, scale. In the case, of the fine-structure constant, though it does run, according to the RG, between the IR (i.e. $m_{e}$ ) and the GUT or Planck scale, this running is relatively small because of the smallness of the factor $(2 \alpha / 3 \pi)$ which multiplies $\ln \left(m_{P} / m_{e}\right)$. As a consequence, one expects that the low-energy EM dilaton coupling $d_{e}$ is similar to its more fundamental high-energy counterpart. [This is also related to the fact that we could neglect, in our action (12) the EM analog of the ratio $\beta_{3}\left(g_{3}\right) / g_{3}$ (i.e. $\beta_{3}\left(g_{3}\right) / g_{3}^{3}$ with geometrically normalized gauge fields), because $\beta_{E M}(e) / e^{3}$ is essentially constant.] The situation is, however, quite different for the low-energy coupling $d_{g}$ to the gluon field energy. There are two equivalent ways of seeing it. One way (used in [15]) precisely consists in drawing the consequences of having a factor $\beta_{3}\left(g_{3}\right) / g_{3}$ in front of $\left(F^{A}\right)^{2}$ (to ensure RG invariance). When comparing the matching of this factor at the Planck scale, versus its meaning at the low-scale $\Lambda_{3} \sim 1 \mathrm{GeV}$, one sees that $d_{g}$ differs from its high-energy counterpart by a largish factor of order

$$
K=f_{\text {h.q. }} \frac{g_{3}\left(\Lambda_{c}\right)}{\beta_{3}\left(\Lambda_{c}\right)}
$$

where the additional factor $f_{\text {h.q. }}$ takes into account the effect of the heavy quarks [15]. The second way (used in [12]) consists in differentiating the expression giving $\Lambda_{3}$ in terms of high-energy boundary conditions. We have seen above that the definition of $\Lambda_{3}$ coming from the integration of the RGrunning equation for $g_{3}$ yields equivalent results, with the same appearance of the largish factor $g_{3}\left(\Lambda_{c}\right) / \beta_{3}\left(\Lambda_{c}\right)$. It is easily checked that this second way also automatically includes the effect of heavy quarks, i.e. the factor $f_{\text {h.q. }}$ in $K$. Actually, this second way provides a quick way to estimate the order of magnitude of the factor $K$ above. Indeed, the reason why $\Lambda_{3}$ is herarchically smaller than $\Lambda_{c}$ is that solving the RG-running equation leads to a result of the type $\Lambda_{3} \sim \Lambda_{c} \exp \left(-C / g_{c}^{2}\right)$. Differentiating this expression w.r.t. $\varphi$ immediately shows that the amplification factor between $d_{g}$ and the high-energy dilaton coupling $\partial \ln g_{c}^{2} / \partial \varphi$ can be written as

$$
K=\ln \left(\Lambda_{c} / \Lambda_{3}\right)
$$

Using, for instance, $\Lambda_{c} \sim m_{P}=1 / \kappa \sim 3.44 \times 10^{18} \mathrm{GeV}$ then yields $K \sim$ $\ln \left(m_{P} / 1 \mathrm{GeV}\right) \sim 42.7$, as in Ref. [12], and consistently with the results of 
[15], for the MSSM case. [We note also that the presence of this logarithmic enhancement factor in the dilaton coupling was pointed out in Ref. [11.]

When considering the low-energy dilaton coupling to the average light quark mass $\hat{m}$, the second way of computing it similarly suggests that it will contain a large enhancement factor $\sim \ln \left(\Lambda_{c} / \hat{m}\right)$ with respect to some high-energy counterpart that should a priori be comparable to $\partial \ln g_{c}^{2} / \partial \varphi$. Indeed, let us recall that the quark masses are of order $m_{q} \sim f H$, where $H$ is the Higgs's VEV, and $f$ a dimensionless Yukawa coupling. As we do not know what is the mechanism which determines (from the UV) the scale of the electroweak breaking (i.e. which allows for a negative squared mass for the Higgs at low energies), we cannot compute the sensitivity of $m_{q}$ to $\varphi$. However, it is plausible, as indicated by the 'no-scale' models [37, that $H$ is related to $\Lambda_{c}$, via the RG-running of (scalar) masses, by an exponential factor similar to the one linking $\Lambda_{3}$ to $\Lambda_{c}$ : more precisely, in these models one has $H \sim \exp \left(-C^{\prime} / h_{t}^{2}\right)$, where $C^{\prime}$ is a constant of order unity, and where $h_{t}$ is the Yukawa coupling of the top quark. Then, the $\varphi$-derivative of $\ln m_{q}$ will also contain an enhancement factor of order $\ln \left(\Lambda_{c} / \Lambda_{3}\right)$, i.e. of the same order as the enhancement $K$ above, but probably differing by a factor of order unity.

Summarizing: it seems theoretically plausible that, starting from dilaton couplings which are of the same order, say $d_{c}=\partial \ln g_{c}^{2} / \partial \varphi$, when considered at the high-energy scale $\Lambda_{c}$, the low-energy coupling EM $d_{e}$ will remain $d_{e} \sim d_{c}$, while $d_{g}$ and the various $d_{m_{a}}$ will be enhanced by factors of order $K_{a} \sim \ln \left(\Lambda_{c} / m_{a}\right) \sim 40$. Notably, we can expect $d_{g} \sim K d_{c}$, and $d_{\hat{m}} \sim K^{\prime} d_{c}$. This leaves us with the problem of estimating the difference $d_{g}-d_{\hat{m}}$ which enters in composition-dependent effects. It is formally of order $\sim\left(K-K^{\prime}\right) d_{c}$. We do not know to what extent there could be a compensation between $K$ and $K^{\prime}$. If such a compensation exists, i.e. if $K-K^{\prime} \sim 1$, instead of $\sim 40$, one will have $d_{g}-d_{\hat{m}} \sim d_{c} \sim d_{e}$. On the other hand, if $K$ and $K^{\prime}$ differ by a factor of order unity (or have a different sign), we will have $d_{g}-d_{\hat{m}} \sim 40 d_{c} \gg d_{e}$. Therefore, we can only write an approximate link of the type $d_{e} \lesssim d_{g}-d_{\hat{m}}$. For our discussion of the relative importance of various EP signals, it would be too restrictive to assume that Nature has chosen the case where $d_{e}$ is significantly smaller than $d_{g}-d_{\hat{m}}$. We shall therefore continue our discussion under the general assumption $d_{e} \sim d_{g}-d_{\hat{m}}$.

\subsection{Simplified parameterization}

Our theoretical treatment of nuclear binding effects has given us some spe-

cific predictions for the numerical coefficients of the various contributions to 
the 'dilaton charges' $Q_{k_{a}}$. To better delineate what they imply for the phenomenology of EP experiments we shall henceforth make some further approximations. First, we replace the overall factor $F_{A}=A m_{\mathrm{amu}} / m_{A}$ by one. This is allowed because we shall see that the leading terms in the $Q_{k_{a}}$ 's vary by factor of a few over the periodic table, while $F_{A}$ differs from one only at the $10^{-3}$ level. The second approximation consists in using the approximate equation (82) to estimate various $Z$-dependent terms in the dilaton charges. Namely, using this link, and taking into account the predicted numerical coefficients in the dilaton charges, one finds that the terms $0.020(A-2 Z)^{2} / A^{2}$ (in $Q_{\hat{m}}$ ), and $0.0017(A-2 Z) / A$ (in $Q_{\delta m}$ ), are numerically subdominant. [We assume here that, e.g., $d_{\delta m}-d_{g} \sim d_{\hat{m}}-d_{g}$ etc.] In addition, we find that we can replace $Z / A$ by $1 / 2$ in $Q_{m_{e}}$ and $Q_{e}$. After these simplifications, we can move some left-over composition-independent numerical coefficients out of the $Q$ 's, and into the general composition-independent contribution $d_{g}$ in $\alpha_{A}$.

After these approximations, we end up with

$$
\alpha_{A} \simeq d_{g}^{*}+\left[\left(d_{\hat{m}}-d_{g}\right) Q_{\hat{m}}^{\prime}+d_{e} Q_{e}^{\prime}\right]_{A}
$$

where

$$
d_{g}^{*}=d_{g}+0.093\left(d_{\hat{m}}-d_{g}\right)+0.00027 d_{e}
$$

and where

$$
Q_{\hat{m}}^{\prime}=-\frac{0.036}{A^{1 / 3}}-1.4 \times 10^{-4} \frac{Z(Z-1)}{A^{4 / 3}}
$$

and

$$
Q_{e}^{\prime}=+7.7 \times 10^{-4} \frac{Z(Z-1)}{A^{4 / 3}} .
$$

We think that these approximate expressions capture all the potentially dominant EP violation effects. We illustrate the variation of these approximate dilaton charges over the periodic table by giving in Table 1 their values for a sample of elements. [Our table considers many of the same elements as Table 1 of [17, but the crucial new information we provide are the numerical factors in the charges, as predicted from our results. We use the (non-integer) atomic weights as an approximate way of averaging 14 the result over the natural isotopic composition. ]

The two main lessons we can draw from Eq. (85) and the numbers in Table 1 are: (i) Contrary to what general phenomenological considerations (of the type of Eq. (81)) could suggest, there are only two dominant EP

\footnotetext{
${ }^{14}$ Essentially we are using the approximation $\langle f(A)\rangle \simeq f(\langle A\rangle)$, which is valid to first order for a smooth function $f(A)$.
} 
Table 1: Approximate EP-violating 'dilaton charges' for a sample of materials. These charges are averaged over the (isotopic or chemical, for $\mathrm{SiO}_{2}$ ) composition.

$\begin{array}{ccccc}\text { Material } & A & Z & -Q_{\hat{m}}^{\prime} & Q_{e}^{\prime} \\ \mathrm{Li} & 7 & 3 & 18.88 \times 10^{-3} & 0.345 \times 10^{-3} \\ \mathrm{Be} & 9 & 4 & 17.40 \times 10^{-3} & 0.494 \times 10^{-3} \\ \mathrm{Al} & 27 & 13 & 12.27 \times 10^{-3} & 1.48 \times 10^{-3} \\ \mathrm{Si} & 28.1 & 14 & 12.1 \times 10^{-3} & 1.64 \times 10^{-3} \\ \mathrm{SiO}_{2} & \ldots & \ldots & 13.39 \times 10^{-3} & 1.34 \times 10^{-3} \\ \mathrm{Ti} & 47.9 & 22 & 10.28 \times 10^{-3} & 2.04 \times 10^{-3} \\ \mathrm{Fe} & 56 & 26 & 9.83 \times 10^{-3} & 2.34 \times 10^{-3} \\ \mathrm{Cu} & 63.6 & 29 & 9.47 \times 10^{-3} & 2.46 \times 10^{-3} \\ \mathrm{Cs} & 133 & 55 & 7.67 \times 10^{-3} & 3.37 \times 10^{-3} \\ \mathrm{Pt} & 195.1 & 78 & 6.95 \times 10^{-3} & 4.09 \times 10^{-3}\end{array}$

violation effects: one, $Q_{e}^{\prime}$, coming from the $\varphi$ sensitivity of the fine-structure constant, and the other one, $Q_{\hat{m}}^{\prime}$, coming from the $\varphi$ sensitivity of the average light quark mass in nuclear binding; (ii) in spite of the seemingly small numerical coefficient entering the $Q_{e}^{\prime}$ term, this term can be comparable to the $Q_{\hat{m}}^{\prime}$ one for heavy elements, such as Platinum or beyond. Actually, one should remember that it is only the variations of the $Q$ 's over the periodic table which matters. From this point of view, note that the total variation of $Q_{\hat{m}}^{\prime}$ between $\mathrm{Li}$ and $\mathrm{Pt}$ is $\sim 10^{-2}$, while the corresponding total variation of $Q_{e}^{\prime}$ is $\sim 4 \times 10^{-3}$. Moreover, while the variation of $Q_{\hat{m}}^{\prime}$ is localized around the light elements, that of $Q_{e}^{\prime}$ keeps increasing for heavy elements. [Formally, $Q_{e}^{\prime} \propto Z^{2} / A^{4 / 3} \sim A^{2 / 3}$, while $Q_{\hat{m}}^{\prime} \propto A^{-1 / 3}$.]

Summarizing: our theoretical framework suggests that there are two dominant 'directions' for the EP-violation signals associated to a long-range dilaton-like field, namely

$$
\left(\frac{\Delta a}{a}\right)_{B C}=\left(\alpha_{B}-\alpha_{C}\right) \alpha_{E}=\left[D_{\hat{m}} Q_{\hat{m}}^{\prime}+D_{e} Q_{e}^{\prime}\right]_{B C}
$$

where $[Q]_{B C} \equiv Q_{B}-Q_{C}$, and where the 'dilaton charges' are (approximately) given by Eq. (73) and Eq. (76). The coefficients $D$ are given by

$$
D_{\hat{m}}=d_{g}^{*}\left(d_{\hat{m}}-d_{g}\right), \quad D_{e}=d_{g}^{*} d_{e}
$$


where

$$
d_{g}^{*} \simeq \alpha^{c . i .} \simeq d_{g}+0.093\left(d_{\hat{m}}-d_{g}\right)
$$

If we were assuming that the dilaton coupling $d_{e}$ is much smaller than $d_{\hat{m}}-d_{g}$, we could go further and conclude (in view of the numerical results indicated in Table I) that the signal $Q_{e}^{\prime}$ is sub-dominant w.r.t. $Q_{\hat{m}}^{\prime}$. In that case we would end up with a uni-dimensional EP signal proportional to $\left[Q_{\hat{m}}^{\prime}\right]_{B C}$.

\subsection{Experimental bounds}

The fact that two types of EP signals are expected to dominate allow one to derive simultaneous constraints on the two dominant theoretical parameters $D_{\hat{m}}, D_{e}$ by using only two independent sets of EP experiments. We can use to that effect the two current EP experiments which have reached the $10^{-13}$ level, namely the terrestrial EötWash experiment, and the celestial Lunar Laser Ranging one

The EötWash collaboration has compared the relative acceleration of Be and $\mathrm{Ti}$ in the gravitational field of the Earth [1]. The Lunar Laser Ranging (LLR) experiments [2] measured the differential acceleration of the Earth and the Moon towards the Sun. We can use our framework to translate the results from these two experiments on constraints on the two theoretical parameters $D_{\hat{m}}, D_{e}$.

The EötWash result concerns $\mathrm{Be}(\mathrm{A}=9, \mathrm{Z}=4)$ and $\mathrm{Ti}(\mathrm{A}=47.9, \mathrm{Z}=22)$, and reads

$$
\left(\frac{\Delta a}{a}\right)_{\mathrm{BeTi}}=\left(\alpha_{B e}-\alpha_{T i}\right) \alpha_{\text {Earth }}=(0.3 \pm 1.8) \times 10^{-13}
$$

Working at the two-sigma level, i.e. $(0.3 \pm 3.6) \times 10^{-13}$, and neglecting the central value 0.3 , the rewriting of this equation in terms of the theoretical parameters $D_{\hat{m}}, D_{e}$ yields

$$
10^{-3}\left[-7.11 D_{\hat{m}}-1.55 D_{e}\right]= \pm 3.6 \times 10^{-13}
$$

The Lunar Laser Ranging measurement constrains the relative acceleration of the Earth and the Moon towards the Sun:

$$
\left(\frac{\Delta a}{a}\right)_{\text {Earth Moon }}=\left(\alpha_{\text {Earth }}-\alpha_{\text {Moon }}\right) \alpha_{\text {Sun }}=(-1.0 \pm 1.4) \times 10^{-13}
$$

In addition to the composition dependence of the matter in these objects, it has the remarkable ability to test the equivalence of the gravitational self 
energy [34]. For dilaton models where the scalar also couples to matter, it is the matter couplings which will be most important 15 , and we will not consider here the gravitational couplings. The Moon has a very similar composition as the Earth's mantle, which is mostly silicate (primarily silicon and oxygen). The composition differences between the Earth and the Moon come primarily from the Earth's core which is dominantly iron.

We approximate the mantle composition as being $\mathrm{SiO}_{2}$, and the Earth' core as being iron. In addition, we follow Ref. [36] in assigning to the core a relative mass of $32 \%$. Working as above at the 2 -sigma level, and rewriting this constraint in terms of our theoretical parameter 16 yields

$$
0.32 \times 10^{-3}\left[3.55 D_{\hat{m}}+1.0 D_{e}\right]= \pm 2.8 \times 10^{-13}
$$

It is interesting to notice the origin of the various numerical coefficients in this equation, as well as in the corresponding EötWash one above. The r.h. sides feature the $10^{-13}$ sensitivity level. The l.h.sides have coefficients of order a few times $10^{-3}$, which is typical for the differences of 'dilaton charges' listed in Table I. In addition, the LLR 1.h.s. has an extra factor 0.32 due to the fact that only $32 \%$ of the Earth differs in composition from the Moon. Finally, we need to solve two linear equations for the two unknowns $D_{\hat{m}}, D_{e}$ and this introduces an inverse determinant which will further increase the result for the $D$ 's. At the end of the day, if one denotes $\epsilon_{E o t}= \pm 3.6 \times 10^{-10}$ and $\epsilon_{L L R}= \pm 2.8 \times 10^{-10}$ (i.e. the two, random two-sigma errors multiplied by $10^{3}$ ) the solution for $D_{\hat{m}}, D_{e}$ reads

$$
\begin{aligned}
D_{\hat{m}} & =-0.625 \epsilon_{E o t}-3.0 \epsilon_{L L R}, \\
D_{e} & =2.2 \epsilon_{E o t}+14.0 \epsilon_{L L R} .
\end{aligned}
$$

If $\epsilon_{E o t}$ and $\epsilon_{L L R}$ were non-zero EP violation signals, this would give us the values of the dilaton parameters in terms of EP data. In the present situation, however, $\epsilon_{E o t}$ and $\epsilon_{L L R}$ are only (independent) random errors. This expression then shows that the LLR error is dominating the error level in the final result. A LLR EP measurement should be about six times

\footnotetext{
${ }^{15}$ Indeed, gravitational self-energy couples to the combination $\eta_{g}=4(\beta-1)-(\gamma-1)$ of post-Newtonian parameters 34]. However, this combination is theoretically predicted [14] to be proportional to $(1-\gamma) / 2 \simeq \alpha^{\text {c.i. }} \sim d_{g}^{2}$ (see above). The fact that the gravitational self energy is a very small fraction of the total mass then allows one to neglect the corresponding effect.

${ }^{16}$ Strictly speaking one should take into account the fact that the EP signal involves slightly different values for the 'external' $\alpha_{E}$, namely the Earth versus the Sun. For simplicity, we use here the (justified) approximation where both are close to the compositionindependent part $d_{g}^{*}$ of $d_{g}$.
} 
below the $10^{-13}$ level to contribute the same error level as a terrestrial EP measurement at the $10^{-13}$ level. Adding the right-hand-sides of the previous expressions in quadrature, finally leads to the following (two-sigma) error levels on our theoretical parameters

$$
D_{\hat{m}}= \pm 0.87 \times 10^{-9}, \quad D_{e}= \pm 4.0 \times 10^{-9}
$$

\subsection{Specific models}

As we discussed above, one expects that a string-theory dilaton (or moduli) will have low-energy couplings to matter of the general form $d_{g} \sim K d_{c}$, $d_{m_{a}}-d_{g} \sim\left(K_{a}-K\right) d_{c}$, and $d_{e} \sim d_{c}$, where $d_{c}$ is some common string-scale dimensionless dilaton coupling, where the enhancement factors $K, K_{a}$ are expected to be comparable and of order 40 , and where $d_{e}$ does not contain any significant enhancement factor. Using the EötWash-LLR-derived constraints given in the preceding section, we then conclude that the string-scale dilaton coupling $d_{c}$ is constrained to be $d_{c}^{2} \lesssim 10^{-9} /\left(K\left|K-K_{\hat{m}}\right|\right) \sim 10^{-12}$.

There are two possible attitudes towards this very stringent constraint. One is to conclude that all the dilaton-like scalar fields of string theory that are massless at tree level must acquire, via loop effects, a large enough mass to make them invisible in current EP experiments (i.e. $m_{\phi}^{-1}<0.2$ $\mathrm{mm}$ ). A second possibility (suggested in [12] ) consists in assuming that loop effects (which depend on the VEV of the dilaton) modify the usual tree-level dilaton dependence $(\propto \exp (-2 \varphi))$ of the various terms entering the string-scale Lagrangian into more complicated functions of $\varphi$, say $B_{i}(\varphi)$, such that these coupling functions reach an extremum at a special value, say $\varphi_{*}$ of $\varphi$. Indeed, under this assumption, Damour and Polyakov [12] have shown that the cosmological evolution of the universe drives the VEV of $\varphi$ towards $\varphi_{*}$, thereby ensuring that the string-scale dilaton coupling $d_{c}$, which is proportional to $\partial \ln g_{c}^{2} / \partial \varphi$, is naturally very small: "Least Coupling Principle" (see also Refs. [38] and [39]). More precisely, [12] showed that, if the extremum is located at a finite field value $\varphi_{*}$, cosmological edvolution would reduce an initial dilaton coupling $d_{c}^{\text {init }}$ by a factor typically 17 of order $F_{t} \sim 10^{-9}$. Taking this attracting factor into account then suggests that the present, late-cosmological-evolution dilaton coupling coefficients are of order

$$
d_{g} \sim d_{g}-d_{m_{a}} \sim 40 d_{e} \sim 4 \times 10^{-8} d_{c}^{\text {init }}
$$

\footnotetext{
${ }^{17}$ We assume here that the curvature parameter $\kappa$ of the dilaton-coupling function $B(\varphi)$ is of order one. See 12 for the $\kappa$ dependence of the total cosmological "attracting factor" $F_{t}(\kappa)$.
} 
If we insert this result into the EP violation deduced from our results above, say

$$
\left(\alpha_{B e}-\alpha_{T i}\right) \alpha_{\text {Earth }} \simeq 7 \times 10^{-3} d_{g}\left(d_{g}-d_{\hat{m}}\right)
$$

we get a rough "prediction" for the level of EP violation of the order

$$
\left(\alpha_{B e}-\alpha_{T i}\right) \alpha_{\text {Earth }} \sim 10^{-17}\left(d_{c}^{\text {init }}\right)^{2},
$$

where $d_{c}^{\text {init }}$ is expected to be of order unity. We note that this result is compatible with the current experimental tests of the EP, but that several planned improved EP experiments [5, 6, 7, 8] will be able to probe this level of EP violation.

In another version of this dilaton-cosmological-attractor mechanism, the attractor point $\varphi_{*}$ is located at infinity in field space ("runaway dilaton" model [13]). This corresponds to dilaton-dependent couplings of the form

$$
B_{i}(\varphi)=C_{i}+b_{i} e^{-\varphi}+\ldots
$$

During the cosmological evolution, the dilaton runs towards (the strongcoupling limit) $\varphi=+\infty$, exponentially suppressing its coupling to matter. Studying the effect of this runaway mechanism during slow-roll inflation allowed Ref. [13] to relate the present value of the composition-independent dilaton coupling $\alpha^{\text {c.i. }} \simeq d_{g}$ to the amplitude $\delta_{H} \sim 5 \times 10^{-5}$ of density fluctuations generated during inflation. This leads to

$$
d_{g} \simeq \alpha_{\text {c.i. }} \sim 3.2 \frac{b_{F}}{c b_{\lambda}} \delta_{H}^{4 /(n+2)}
$$

where $n$ denotes the power of the inflaton $\chi$ in the inflationary potential, $V(\chi) \propto \chi^{n}$. For instance, in the case of the simplest inflationary potential $V(\chi)=\frac{1}{2} m_{\chi}^{2} \chi^{2}$, i.e. $n=2$, the above result leads to

$$
d_{g}^{2} \sim 2.5 \times 10^{-8}\left(\frac{b_{F}}{c b_{\lambda}}\right)^{2}
$$

In view of our present new results, Eq. (99), on the level of EP violation associated to such a composition-independent coupling, this corresponds to

$$
\left(\alpha_{B e}-\alpha_{T i}\right) \alpha_{\text {Earth }} \sim 2 \times 10^{-10}\left(\frac{b_{F}}{c b_{\lambda}}\right)^{2}
$$

This is in conflict with the current EP tests, except if one assumes that the combination of dimensionless parameters $b_{F} /\left(c b_{\lambda}\right)$ (which was assumed in 
13 to be of order unity) happens to be smaller than about $1 / 30$. In such a model, one would expect to see EP violations just below the currently tested level. Alternatively, one might interpret the constraint from current EP tests as suggesting that the (effective) power of the inflaton in the inflationary potential $V(\chi)$ is less than $n=2$. For instance, if $n \approx 0$, Eq. (102) implies $d_{g}^{2} \simeq 6 \times 10^{-17}$, corresponding to $\Delta a / a \sim 4 \times 10^{-19}$.

Finally, a recent work [40] suggests the existence of couplings of a light scalar which are quite different from the usual string-motivated ones. In the model of Ref. [40] the light scalar couples only to quark mass terms, through mixing with the Higgs. At tree level, the couplings are

$$
d_{m i}=\frac{A}{\kappa m_{H}^{2}}
$$

where $A$ is a very small mixing parameter and $m_{H}$ is the mass of the Higgs boson. However, integrating out the heavy (t,b,c) quarks (à la [18, 15]) induces gluonic couplings

$$
d_{g}=\frac{2 A}{9 \kappa m_{H}^{2}}
$$

The constraint of this model can be then calculated to be

$$
\left[\frac{A}{\kappa m_{H}^{2}}\right]^{2}<4.0 \times 10^{-10} \text {. }
$$

\section{Experimental sensitivities}

It can be useful to use a well-motivated parameterized theoretical model as a guideline for comparing the significance, and relative sensitivities, of different experiments. For instance, the parametrized post-Newtonian framework [41] played a useful role in comparing the theoretical significance of various composition-independent tests of relativistic gravity. Here, we wish to capitalize on the better understanding, explained above, of the coupling of a generic dilaton-like field to nuclear binding energy to propose such parametrized frameworks for comparing different compositiondependent tests of gravity. Our proposal is intended as an update, or a specification, of previous similar proposals (see, e.g. [12, 17]). Actually, our proposal is two-headed.

On the one hand, if we make minimal assumptions, and essentially no approximations, we propose to parameterize EP violations by means of the matter coupling (71), which involves five parameters. One of 
them, $d_{g}$ (or more accurately $d_{g}^{*}=\left\langle\alpha_{A}\right\rangle$ ) measures the compositionindependent part of the matter coupling, and can, in principle, be measured by composition-independent gravity tests. The other four parameters, $d_{\hat{m}}-d_{g}, d_{\delta m}-d_{g}, d_{m_{e}}-d_{g}, d_{e}$ are associated with four different types of EP-violation signals, associated to the four different 'dilaton charges' $Q_{\hat{m}}, Q_{\delta m}, Q_{m_{e}}, Q_{e}$, defined in Eqs. (73), (74), (75), and (76).

On the other hand, we have pointed out that two 'directions' of EP violations are likely to dominate the measured signals. They correspond to the two charges $Q_{\hat{m}}$ and $Q_{e}$, i.e. to the two dilaton parameters $d_{\hat{m}}-d_{g}$ and $d_{e}$. For brevity we shall denote the first one as

$$
d_{q} \equiv d_{\hat{m}}-d_{g}
$$

It measures the dilaton coupling to the ratio $\hat{m} / \Lambda_{3}$ of the average light-quark mass to the QCD scale. We recall that the second one, $d_{e}$ is associated to the $\varphi$ sensitivity of the fine-structure constant $\alpha=e^{2} /(4 \pi)$. In the same approximation that these charges dominate, we can simplify the expression of the the matter coupling $\alpha_{A}$ and the corresponding charges, see Eq. (85), and the equations following it. The latter, simplified two-EP-parameter framework 18 is quite predictive, and could be useful as a guideline for comparing and/or planning EP experiments. Let us briefly indicate some consequences of our proposals.

\subsection{Composition independent constraints}

The first useful result in the simplified "reference dilaton model" is the expected ratio between composition-independent effects and compositiondependent ones. As explained above the former are essentially measured by the Eddington parameter 19

$$
1-\gamma \simeq 2 d_{g}^{2}
$$

\footnotetext{
${ }^{18}$ In all, this model contains three independent parameters: $d_{g}, d_{q}$ and $d_{e}$. If one could argue that the $\varphi$ sensitivity of $\kappa \hat{m}$ is much smaller than that of $\kappa \Lambda_{3}$ one could even consider a much more special one-parameter guideline model keeping only $d_{g}$ and setting to zero the various mass couplings $d_{m_{a}}$ as well as $d_{e}$. In such a model $d_{q}=-d_{g}$ would be fixed in terms of $d_{g}$. However, the no-scale supergravity models (and their string realizations) rather suggest that the $d_{m_{a}}$ 's contain logarithmic amplification factors which are comparable to the one expected to be present in $d_{g}$.

${ }^{19}$ Here $d_{g}$ should more accurately be replaced by some average $\left\langle\alpha_{A}\right\rangle \equiv d_{g}^{*}=d_{g}+c d_{q}$, with a coefficient $c \sim 0.1$ depending of the average composition of the considered source bodies.
} 
while the latter are given, say, by Eq. (99). Note that the numerical value $7 \times 10^{-3}$ in the latter equation comes from the $Q_{\hat{m}}^{\prime}$ charge difference between $\mathrm{Be}$ and $\mathrm{Ti}$. We can use instead the maximal difference of $10^{-2}$ corresponding to Be and Pt. This yields the approximate link

$$
\frac{\Delta a}{a} \sim 10^{-2} \frac{d_{q}}{d_{g}} \frac{1-\gamma}{2}
$$

Note that, assuming $d_{q} \sim d_{g}$, this differs by two orders of magnitude from the link $\Delta a / a \sim 10^{-4}(1-\gamma) / 2$ estimated in [12] from considering as dominant the EM coupling $d_{e}$ instead of $d_{q}$. This suggests that current EP tests correspond to post-Newtonian tests at the level $(1-\gamma) / 2 \sim 10^{-11}$, i.e. six orders of magnitude below the current best post-Newtonian test, namely the Cassini limit Eq. (78). [Using the results derived above from combining Eotwash and LLR data, one actually gets a constraint at the level $(1-\gamma) / 2 \sim$ $10^{-9}$, where the loss of a factor 100 comes from the combination of effects explained above.]

\subsection{Test materials}

Concerning the comparison among the sensitivities of different EP experiments, we already gave above an example of the use of our framework (comparison between Eotwash and LLR). Let us also mention another illustrative example. Note that each EP comparison of a pair of materials, say $(B, C)$, corresponds, within our simplified framework, to looking for a signal of the form $\mathbf{D} \cdot \mathbf{Q}_{B C}$, where $\mathbf{D}$ is the two-dimensional vector of dilaton couplings $\left(D_{\hat{m}}, D_{e}\right)$, and $\mathbf{Q}$ a two-dimensional vector of dilaton-charge differences $\left(Q_{\hat{m}}^{\prime}, Q_{e}^{\prime}\right)_{B C}=\left(Q_{\hat{m}}^{\prime}, Q_{e}^{\prime}\right)_{B}-\left(Q_{\hat{m}}^{\prime}, Q_{e}^{\prime}\right)_{C}$. For instance, the current best Eotwash comparison concerned Be and Ti, i.e. (using Table 1) the 'charge' vector $\mathbf{Q}_{T i B e}=(7.11,1.55) \times 10^{-3}$. By contrast, the MICROSCOPE experiment plans to use a pair $\mathrm{Ti}$, Pt, which corresponds to the charge vector $\mathbf{Q}_{P t T i}=(3.33,2.04) \times 10^{-3}$. We see that the two choices are nicely complementary in that the former (using lighter elements) gives more weight to the $\hat{m}$ component of the EP violation, while the latter (with heavier elements) gives approximately equal weights to the $\hat{m}$ and $e$ directions.

\subsection{Atomic interferometry}

Special mention should be given to the sensitivity of EP experiments based on atomic-interferometer techniques. For instance, Ref. [8 mentions the possibility of comparing two isotopes of Rubidium: $\left({ }^{85} \mathrm{Rb},{ }^{87} \mathrm{Rb}\right)$. In such 
a case, we wish to warn the reader that one should not blindly use the formulas that we have derived above, especially the approximate ones for $\left(Q_{\hat{m}}^{\prime}, Q_{e}^{\prime}\right)$. Indeed, the approximations used to simplify the charges employed the average link (82) between $Z$ and $A$. This approximation is acceptable if one compares elements that are distant along the periodic table, but is definitely invalid for isotopes of the same $Z$. Therefore, one should start from our original, non-approximated expressions for the charges.

The use of our ('exact') dilaton charges, suggests that an EP test comparing $\left({ }^{85} \mathrm{Rb},{ }^{87} \mathrm{Rb}\right)$ would correspond, in the full four-dimensional space of $\left(\hat{m}, \delta m, m_{e}, e\right)$, to a charge vector equal to $\mathbf{Q}_{87} R b^{85} R b=$ $(-3.3,3.4,-0.55,-9.2) \times 10^{-5}$. Note that the components of this vector are significantly smaller than those of the charge vectors probed by the other experiments. The dominant direction is along $e$. Note also that the $\delta m$ direction now plays a role as significant as the $\hat{m}$ one, because, besides the binding energies, a crucial effect in comparing two isotopes is evidently a change in the number of neutrons. This also shows that such experiments are complementary to the usual ones, in that they probe new directions in theory space, though it comes at the cost of the overall sensitivity.

The atomic interferometer proposal of [9] suggests the comparison of ${ }^{7} \mathrm{Li}$ and ${ }^{133} \mathrm{Cs}$ atoms. In contrast to the Rubidium experiment, these elements are well separated in $A, Z$, and our simplified charges can be used. We find that this comparison is quite sensitive to the dilaton couplings with dilaton charge vector $\mathbf{Q}_{C s L i}=(11.2,3.02) \times 10^{-3}$. While the experimental comparison of dissimilar atoms may be more difficult than the use of related isotopes, the sensitivity to the dilaton couplings is much increased.

Let us also make some further comments relevant for comparing two isotopes which are very close in mass. Our derivation assumed that the semi-empirical mass formula was an accurate representation of the binding energies. However, this mass formula is an average, which does not always accurately capture local fluctuations, and notably fluctuations linked to varying $A$ for a fixed $Z$. In addition, our derivation has neglected the pairing term $-\delta a_{p} / A^{1 / 2}$, as being subdominant. However, this term might become very important if one were to compare isotopes with mass numbers $A$ differing by an odd integer. Indeed, in that case $\delta=\frac{1}{2}\left[(-)^{N}+(-)^{Z}\right]$ changes by one unit between the two isotopes, and therefore yields a full contribution $a_{p}$ to their mass difference, and thereby also to the dilaton sensitivity. Actually, we would suggest to try to take advantage of this fact by using such odd-related isotopes which are likely to have an enhanced sensitivity to EP violations. [We are aware, however, that this proposal poses both theoretical challenges (determining the $\varphi$ sensitivity of $a_{p}$ ), and experimental ones 
(as the two isotopes will have a different Fermi/Bose statistics, which might undermine the possibility of using accurate, Bose-Einstein-Condensationbased, techniques).]

\subsection{Other applications}

Let us also mention that our framework can be straightforwardly applied to comparing (weak) equivalence principle tests to atomic-clock tests of the dependence of coupling constants on the gravitational potential. The link between these two types of tests has been discussed by several authors [41, 42, 43, 17]. Let us indicate how it is formulated in our notation. The spacetime dependence of the dilaton field is approximately of the form: $\varphi(x, t)=\varphi_{0}(t)+\varphi_{\text {loc }}(x, t)$, where $\varphi_{0}(t)$ is the cosmological value of $\varphi$, and where

$$
\varphi_{\mathrm{loc}}(x, t)=-\sum_{E} \alpha_{E} \frac{G m_{E}}{r_{E}} \simeq-\alpha^{\mathrm{c} . \mathrm{i} .} U(x, t)
$$

gives the influence of the local matter distribution, in terms of the local gravitational potential $U(U>0)$. In the second expression, we have used the approximation $\alpha_{E} \simeq \alpha^{\text {c.i. }}=d_{g}^{*}$. Combining this result with our parametrization $k_{a}(\varphi)=\left(1+d_{a} \varphi\right) k_{a}(0)$ of the $\varphi$ dependence of the various constants $k_{a}=\hat{m} / \Lambda_{3}, \delta m / \Lambda_{3}, m_{e} / \Lambda_{3}, \alpha=e^{2} /(4 \pi)$, we see that the local gravitational potential influences the values of the constants $k_{a}$ measured, say, on the Earth, according to

$$
k_{a}^{\text {loc }}=\left(1-D_{a} U\right) k_{a}\left(\varphi_{0}(t)\right)
$$

where the coefficients $D_{a} \equiv d_{a} \alpha^{\text {c.i. }}=d_{a} d_{g}^{*}$, i.e. $D_{\hat{m}}=d_{g}^{*}\left(d_{\hat{m}}-d_{g}\right), \ldots, D_{e}=$ $d_{g}^{*} d_{e}$ are the same dilaton coefficients that entered our discussion above of the EP tests. Then, to compute the effect of the seasonally varying $U$ on, say, the frequencies of atomic clocks, one needs to know the sensitivity of these frequencies to variations in the $k_{a}$ 's (see [44]). In particular, the $D_{e}= \pm 4 \times 10^{-9}$ two-sigma bound derived above on $D_{e}$, combined with the yearly variation $\Delta U \simeq 3 \times 10^{-10}$ linked with the Earth's eccentricity, shows that EP tests constrain the yearly variation of the fine-structure constant on the Earth to be smaller than $1.2 \times 10^{-18}$ (two sigma). This is about 40 times smaller than the current best atomic-clock experimental sensitivity to the variation of $\alpha$ [45]. Note, however, that clock-comparison experiments are sensitive to different combinations of the parameters $D_{a}$ than EP tests 42. We shall not discuss here the cosmological aspects of the variation of constants, which are more model-dependent. For instance, in the context of the dilaton-runaway model, one can relate the present rate of variation of 
the 'constants' to the (square root of the) EP violation level, see Eq. (3.25) of [13].

Let us finally remark that it would be interesting to use the recent progress (reported in [21, 22] and here) about the quark-mass dependence of nuclear binding to try to derive a well-justified estimate of the quarkmass dependence of the crucial very low-energy neutron capture resonance $E_{r} \simeq 0.1 \mathrm{eV}=10^{-7} \mathrm{MeV}$ of ${ }^{149} \mathrm{Sm}$. Indeed, the analysis of the Oklo data [46, 47, 48] shows that this resonance has not changed by more than about $0.1 \mathrm{eV}$ since the Oklo natural fission reactor was in activity 2 billion years ago. A naive use of our results, based on our finding that the bulk binding energy per nucleon, $a_{v}$, varies with $\hat{m}$ as $\Delta a_{v} \simeq-42 \Delta \ln \hat{m} / \Lambda_{3} \mathrm{MeV}$, suggests that Oklo data constrain the fractional variation of $\hat{m} / \Lambda_{3}$ over 2 billion years to the level $\Delta \ln \hat{m} / \Lambda_{3} \lesssim 10^{-7} / 42 \sim 2.4 \times 10^{-9}$. Such a limit would be a very significant constraint on the possible cosmological evolution of the dilaton. However, it is not clear whether a detailed study of the specific (unstable) energy level corresponding to $E_{r}$ will confirm this sensitivity to $\hat{m} / \Lambda_{3}$.

\section{Conclusions}

We have provided a parametrized framework for the study of the equivalence principle 20 in models with light, dilaton-like scalar particles. Our general framework contains five independent parameters, and should be applicable to the low-energy limit of many models. The most novel aspect of our work was to provide an estimate of the effects of the dilaton coupling to nuclear binding energy. We have found that these couplings induce, as leading effect, equivalence principle violations varying with the mass number as $A^{-1 / 3}$. The level of these EP violations is expected to be at least comparable to (and, for lighter elements, somewhat larger than) that associated to the Coulomb energy.

We have also provided a simplified scalar model, containing three parameters: one composition-independent parameter, and two compositiondependent ones. This model is expected to describe the dominant effects of the most general 5-parameter framework. We suggest to use it as a guideline for comparing and planning EP experiments. We used it to combine

\footnotetext{
${ }^{20}$ Here we have limited our considerations to the weak equivalence principle (tests of the universality of free fall). However, our parametrized Lagrangian can also be used to study the effect of dilaton couplings on other aspects of the EP: such has clock-comparison experiments.
} 
Eötvos and Lunar Laser Ranging data so as to constrain its two theoretical composition-dependent parameters. We found that they are constrained at the $10^{-9}$ level. This plausibly implies (in our model, and using some naturality assumption) a corresponding limit on composition-independent effects at about the same level, i.e. $(1-\gamma) / 2 \lesssim 10^{-9}$, which is four orders of magnitude below the best present composition-independent gravitational tests (Cassini experiment).

In the happy future situation of several non-zero measurements of EP violations, one could check the consistency of our simplified model, which is quite predictive. If needed the other scalar couplings could readily be included to make sense of subleading effects modifying the simple predictions of this simplified model.

\section{Acknowledgements}

JFD thanks the IHES for hospitality both at the start of this project and at its conclusion. He also acknowledges support partially by the NSF grants PHY- 055304 and PHY - 0855119, and in part by the Foundational Questions Institute. We thank Ulf Meißner for a useful correspondence.

\section{Appendix: The strange quark mass}

We are not able to provide a definitive calculation of how equivalence principle violations depend on the strange quark couplings. This is an area where there is no consensus and the motifs of the day change quickly. While we cannot solve this issue, we will here argue that the the strange quark dependence could be about or within the uncertainty that we are quoting.

When quarks are heavy, they can be integrated out with the result simply going into a modification of the gluonic coupling, $d_{g}$. The $u, d$ quarks are light, are directly involved in nucleon couplings and are clearly active dynamically in nucleon binding. The strange quark is intermediate in mass. Nucleons do not explicitly contain strange quarks, so their effects are secondary. Certainly they couple to nucleons at some level through loop effects. Initial theoretical calculations suggested that these couplings could be quite large. However, increasingly theoretical and experimental developments are bounding these effects to be relatively small.

Fortunately for equivalence principle violations, the leading manifestations of the strange quark mass would not have an effect in any case. For example, the much debated contribution of the strange quark to the mass of 
neutrons and protons [49] would not lead to the violation of the equivalence principle. This is because the effect is an isospin singlet and contributes equally to the neutron and proton, so that the total effect in an atomic state is proportional to $A$. This leads to a constant contribution to $\alpha_{A}$ independent of $A$, and no violation of the equivalence principle. Note that the large effects suggested for strange contributions to nucleon masses recently have been bounded by lattice computations to be consistent with zero [50]. In nuclear binding, the leading $A$ dependent term does not violate the equivalence principle, and it is only the surface term that is relevant. Therefore the key feature to be estimated is the strange quark contribution to the surface binding energy.

In discussing the binding energy it is easy to be led astray. For example early estimates used kaon loops in chiral perturbation theory to conclude that there was a very large effect [51. However, it has become clear from dispersive work, such as our own, that the $\bar{K} K$ intermediate state enters above the region of validity of chiral calculations [52]. There are analytic studies that show that the reliable low energy portions from such loops are very small [53, and lattice studies have definitively shown that the chiral loop effects are not strongly present at such large masses [54].

The lightest intermediate states involving strangeness that can couple to nucleons are that of a $K \bar{K}$ intermediate state and also the vector $\varphi$ meson (an $\bar{s} s$ bound state, not to be confused with our notation for the dilaton). In dispersive treatments, both of these start at $1 \mathrm{GeV}$. The coupling of the $\varphi$ to nucleons is highly uncertain, and depends more on the assumptions made in a given calculation than in a unique piece of evidence in its favor. Moreover it is highly constrained by recent experiments [55] that show smaller than expected hidden strange couplings in nucleons. If we use a estimate which we find to be reasonable and which is within the constraints of present experiments [56], the $\varphi$ effects are too small to be significant.

However, $K \bar{K}$ intermediate states can contribute to the leading scalar interaction and may have a non-trivial effect. We expect from most models of the nuclear potential that most of the scalar strength comes from below 1 $\mathrm{GeV}$. The effect of $M \bar{M}$ intermediate states must decouple as the mass of the meson $M$ gets large. If we estimate generously that $K \bar{K}$ intermediate states contributes $10-15 \%$ to the scalar strength, and we take a typical form factor to account for the high mass threshold of the form $\left(\Lambda^{2}+4 m_{K}^{2}\right)^{-1}$ (where $\Lambda$ is is some typical form factor scale), we would estimate the strange quark 
mass dependence

$$
m_{s} \frac{\partial \eta_{S}}{\partial m_{s}}=m_{K}^{2} \frac{\partial \eta_{S}}{\partial m_{K}^{2}}=(0.10-0.15) \frac{4 m_{K}^{2}}{\Lambda^{2}+4 m_{K}^{2}} \sim 0.07-0.10
$$

using $\Lambda^{2}=m_{\rho}^{2}$. Comparison with Eq. (49) indicates that this is comparable to the error bar that we assigned to that calculation. If the $K \bar{K}$ is positive as expected, a contribution of this size could lead to a 20-30\% increase in the coefficient of the leading $A^{-1 / 3}$ term in our final results. This is clearly a crude estimate, but we don't expect that it is grossly misleading.

\section{References}

[1] S. Schlamminger, K. Y. Choi, T. A. Wagner, J. H. Gundlach and E. G. Adelberger, "Test of the Equivalence Principle Using a Rotating Torsion Balance," Phys. Rev. Lett. 100, 041101 (2008) arXiv:0712.0607 [gr-qc]].

[2] J. G. Williams, S. G. Turyshev and D. H. Boggs, "Progress in Lunar Laser Ranging Tests of Relativistic Gravity," Phys. Rev. Lett. 93, 261101 (2004) arXiv:gr-qc/0411113. J. G. Williams, S. G. Turyshev and D. H. Boggs, "Lunar Laser Ranging Tests of the Equivalence Principle with the Earth and Moon," Int. J. Mod. Phys. D 18, 1129 (2009) arXiv:gr-qc/0507083.

[3] T. Damour, "Questioning the equivalence principle," arXiv:gr-qc/0109063.

T. Damour, "Testing the equivalence principle: Why and how?," Class. Quant. Grav. 13, A33 (1996) arXiv:gr-qc/9606080. T. Damour, J.P. Blaser; "Optimizing the choice of materials in equivalence principle experiments" in Particle Astrophysics, Atomic Physics and Gravitation, Proceedings of the XIVth Moriond Workshop, edited by J. Tran Than Van, G. Fontaine and E. Hinds, Editions Frontires, Gif-sur-Yvette, pp 433-440, 1994.

[4] E. G. Adelberger et al., "Opportunities for Probing Fundamental Gravity with Solar System Experiments," arXiv:0902.3004 [gr-qc].

[5] http://smsc.cnes.fr/MICROSCOPE/index.htm

[6] http://eotvos.dm.unipi.it/ 
[7] http://www.sstd.rl.ac.uk/fundphys/step/

http://einstein.stanford.edu/STEP/index.html

[8] S. Dimopoulos, P. W. Graham, J. M. Hogan and M. A. Kasevich, "Testing General Relativity with Atom Interferometry," Phys. Rev. Lett. 98, 111102 (2007) arXiv:gr-qc/0610047.

S. Dimopoulos, P. W. Graham, J. M. Hogan and M. A. Kasevich, "General Relativistic Effects in Atom Interferometry," Phys. Rev. D 78, 042003 (2008) arXiv:0802.4098 [hep-ph]].

[9] G. Kim and H. Mueller, "Test of the equivalence principle using Li atom interferometry", Bull. of the American Physical Society Vol. 55, No 5, E1.00009 (2010)

[10] R. D. Reasenberg and J. D. Phillips, "A weak equivalence principle test on a suborbital rocket," Class. Quant. Grav. 27 (2010) 095005 arXiv:1001.4752 [gr-qc]].

[11] T. R. Taylor and G. Veneziano, "Dilaton Couplings at Large Distances," Phys. Lett. B 213, 450 (1988).

[12] T. Damour and A. M. Polyakov, "String theory and gravity," Gen. Rel. Grav. 26, 1171 (1994) arXiv:gr-qc/9411069.

T. Damour and A. M. Polyakov, "The String Dilaton And A Least Coupling Principle," Nucl. Phys. B 423, 532 (1994) [arXiv:hep-th/9401069].

[13] T. Damour, F. Piazza and G. Veneziano, "Runaway dilaton and equivalence principle violations," Phys. Rev. Lett. 89, 081601 (2002) arXiv:gr-qc/0204094.

T. Damour, F. Piazza and G. Veneziano, "Violations of the equivalence principle in a dilaton-runaway scenario," Phys. Rev. D 66, 046007 (2002) arXiv:hep-th/0205111.

[14] T. Damour and G. Esposito-Farese, "Tensor multiscalar theories of gravitation," Class. Quant. Grav. 9, 2093 (1992).

[15] D. B. Kaplan and M. B. Wise, "Couplings of a light dilaton and violations of the equivalence principle," JHEP 0008, 037 (2000) arXiv:hep-ph/0008116.

[16] T. Damour and J. F. Donoghue, "Phenomenology of the equivalence principle with light scalars", arXiv:1007.2790 [gr-qc]. 
[17] T. Dent, "Eotvos bounds on couplings of fundamental parameters to gravity," Phys. Rev. Lett. 101, 041102 (2008) arXiv:0805.0318 [hep$\mathrm{ph}]$.

[18] M. A. Shifman, A. I. Vainshtein and V. I. Zakharov, "Remarks On Higgs Boson Interactions With Nucleons," Phys. Lett. B 78, 443 (1978).

[19] J. C. Collins, A. Duncan and S. D. Joglekar, "Trace And Dilatation Anomalies In Gauge Theories," Phys. Rev. D 16, 438 (1977).

[20] C. Amsler et al. (Particle Data Group), Physics Letters B667, 1 (2008) and 2009 partial update for the 2010 edition

[21] J. F. Donoghue, "The Nuclear Central Force in the Chiral Limit," Phys. Rev. C 74, 024002 (2006) arXiv:nucl-th/0603016.

[22] T. Damour and J. F. Donoghue, "Constraints on the variability of quark masses from nuclear binding," Phys. Rev. D 78, 014014 (2008) [arXiv:0712.2968 [hep-ph]].

[23] P. E. Hodgson, E. Gadioli and E. Gadioli Erba, "Introductory nuclear physics," Oxford, UK: Clarendon (1997) $723 p$

[24] R. J. Furnstahl and B. D. Serot, "Parameter Counting in Relativistic Mean-Field Models," Nucl. Phys. A 671, 447 (2000) [arXiv:nucl-th/9911019].

[25] B. A Nilolaus, T. Hoch and D. G. Madland, "Nuclear ground state properties in a relativistic point coupling model", Phys. Rev. C46, 1757 (1992)

B. D. Serot and J. D. Walecka, "Effective field theory in nuclear manybody physics," arXiv:nucl-th/0010031.

J. J. Rusnak and R. J. Furnstahl, "Relativistic point-coupling models as effective theories of nuclei," Nucl. Phys. A 627, 495 (1997) arXiv:nucl-th/9708040.

B. Machleidt and D. R. Entem, "Towards a consistent approach to nuclear structure: EFT of two- and many-body forces" arXive:nucl-th/0503025

[26] J. F. Donoghue, "Sigma exchange in the nuclear force and effective field theory," Phys. Lett. B 643, 165 (2006) arXiv:nucl-th/0602074. 
[27] S. R. Beane and M. J. Savage, "Variation of fundamental couplings and nuclear forces," Nucl. Phys. A 713, 148 (2003) arXiv:hep-ph/0206113]. S. R. Beane and M. J. Savage, "The quark mass dependence of two-nucleon systems," Nucl. Phys. A 717, 91 (2003) arXiv:nucl-th/0208021.

E. Epelbaum, U. G. Meissner and W. Gloeckle, "Further comments on nuclear forces in the chiral limit," arXiv:nucl-th/0208040. V. V. Flambaum and R. B. Wiringa, "Dependence of nuclear binding on hadronic mass variation," Phys. Rev. C 76, 054002 (2007) arXiv:0709.0077 [nucl-th]].

[28] E. Epelbaum, W. Glockle, A. Kruger and U. G. Meissner, "Effective theory for the two-nucleon system," Nucl. Phys. A 645, 413 (1999) arXiv:nucl-th/9809084.

M. J. Savage, "Effective field theory for nuclear physics," arXiv:nucl-th/0301058.

P. F. Bedaque and U. van Kolck, "Effective field theory for fewnucleon systems," Ann. Rev. Nucl. Part. Sci. 52, 339 (2002) arXiv:nucl-th/0203055.

[29] E. Epelbaum, W. Glöckle and U. G. Meißner, "Improving the convergence of the chiral expansion for nuclear forces. I: Peripheral phases," arXiv:nucl-th/0304037.

E. Epelbaum, W. Gloeckle and U. G. Meißner, "Improving the convergence of the chiral expansion for nuclear forces. II: Low phases and the deuteron," Eur. Phys. J. A 19, 401 (2004) arXiv:nucl-th/0308010.

N. Kaiser, "Chiral $2 \pi$ exchange N N potentials: Two-loop contributions," Phys. Rev. C 64, 057001 (2001) arXiv:nucl-th/0107064.

D. R. Entem and R. Machleidt, "Chiral $2 \pi$ exchange at order four and peripheral N N scattering," Phys. Rev. C 66, 014002 (2002) arXiv:nucl-th/0202039. N. Kaiser, "Chiral $2 \pi$-exchange N N potentials: Relativistic 1/M ${ }^{2}$-corrections," Phys. Rev. C 65, 017001 (2002) [arXiv:nucl-th/0109071].

N. Kaiser, "Chiral $3 \pi$ exchange $\mathrm{N}$ potentials: Results for representation-invariant classes of diagrams," Phys. Rev. C 61, 014003 (2000) [arXiv:nucl-th/9910044].

$\mathrm{N}$. Kaiser, "Chiral $3 \pi$ exchange $\mathrm{N} N$ potentials: Results for diagrams proportional to $g_{A}^{4}$ and $g_{A}^{6}$," Phys. Rev. C 62, 024001 (2000) arXiv:nucl-th/9912054.

N. Kaiser, "Chiral $3 \pi$-exchange N N potentials: Results for dominant 
next-to-leading order contributions," Phys. Rev. C 63, 044010 (2001) arXiv:nucl-th/0101052.

[30] B. D. Serot and J. D. Walecka, "Recent progress in quantum hadrodynamics," Int. J. Mod. Phys. E 6, 515 (1997) [arXiv:nucl-th/9701058].

[31] J. Gasser and H. Leutwyler, "Quark Masses," Phys. Rept. 87, 77 (1982).

[32] B. Bertotti, L. Iess and P. Tortora, "A test of general relativity using radio links with the Cassini spacecraft," Nature 425 (2003) 374.

[33] J. Gasser, H. Leutwyler and M. E. Sainio, "Sigma term update," Phys. Lett. B 253, 252 (1991).

[34] K. Nordtvedt, "Testing relativity with laser ranging to the moon," Phys. Rev. 170, 1186 (1968).

[35] D.L. Anderson, Theory of the Earth (Blackwell Scientific Publications, Oxford, 1989), p.18, cited in S. Baessler, B. R. Heckel, E. G. Adelberger, J. H. Gundlach, U. Schmidt and H. E. Swanson, "Improved Test of the Equivalence Principle for Gravitational Self-Energy," Phys. Rev. Lett. 83, 3585 (1999).

[36] T. Damour and D. Vokrouhlicky, "The Equivalence Principle And The Moon," Phys. Rev. D 53, 4177 (1996) arXiv:gr-qc/9507016].

[37] J. R. Ellis, C. Kounnas and D. V. Nanopoulos, "No Scale Supersymmetric Guts," Nucl. Phys. B 247, 373 (1984).

[38] T. Damour and K. Nordtvedt, "General relativity as a cosmological attractor of tensor scalar theories," Phys. Rev. Lett. 70, 2217 (1993); T. Damour and K. Nordtvedt, and "Tensor - scalar cosmological models and their relaxation toward general relativity," Phys. Rev. D 48, 3436 (1993).

[39] P. Brax, C. van de Bruck, A. C. Davis and D. J. Shaw, "The Dilaton and Modified Gravity," arXiv:1005.3735 [astro-ph.CO].

[40] F. Piazza and M. Pospelov, "Sub-eV scalar dark matter through the super-renormalizable Higgs portal," arXiv:1003.2313 [hep-ph].

[41] C. M. Will, "The confrontation between general relativity and experiment," Living Rev. Rel. 9, 3 (2005) arXiv:gr-qc/0510072. 
[42] T. Damour, "Gravity, equivalence principle and clocks," arXiv:gr-qc/9711060; "Equivalence principle and clocks," arXiv:gr-qc/9904032,

[43] K. Nordtvedt, "Space-time variation of physical constants and the equivalence principle," Int. J. Mod. Phys. A 17, 2711 (2002).

[44] V. V. Flambaum and A. F. Tedesco, "Dependence of nuclear magnetic moments on quark masses and limits on temporal variation of fundamental constants from atomic clock experiments," Phys. Rev. C 73, 055501 (2006) arXiv:nucl-th/0601050.

[45] T. Rosenband et al. Frequency Ratio of Al+ and Hg+ Single-Ion Optical Clocks; Metrology at the 17th Decimal Place Science 319, 1808 (2008). [DOI: 10.1126/science.1154622]

[46] T. Damour and F. Dyson, "The Oklo bound on the time variation of the fine-structure constant revisited," Nucl. Phys. B 480, 37 (1996) arXiv:hep-ph/9606486.

[47] C. R. Gould, E. I. Sharapov and S. K. Lamoreaux, "Time-variability of alpha from realistic models of Oklo reactors," Phys. Rev. C 74, 024607 (2006) arXiv:nucl-ex/0701019.

[48] Yu. V. Petrov, A. I. Nazarov, M. S. Onegin, V. Y. Petrov and E. G. Sakhnovsky, "Natural nuclear reactor Oklo and variation of fundamental constants. I: Computation of neutronic of fresh core," Phys. Rev. C 74, 064610 (2006) arXiv:hep-ph/0506186].

[49] C. A. Dominguez and P. Langacker, "Present Status Of The Pion Nucleon Sigma Term," Phys. Rev. D 24, 1905 (1981).

J. F. Donoghue and C. R. Nappi, "The Quark Content Of The Proton," Phys. Lett. B 168, 105 (1986).

R. L. Jaffe and C. L. Korpa, "The Pattern of Chiral Symmetry Breaking and the Strange Quark Content of the Proton," Comments Nucl. Part. Phys. 17, 163 (1987).

[50] K. Takeda, S. Aoki, S. Hashimoto, T. Kaneko, T. Onogi and N. Yamada [JLQCD collaboration], "Calculation of nucleon strange quark content with dynamical overlap quarks," arXiv:0910.5036 [hep-lat].

H. Ohki et al., "Nucleon sigma term and strange quark content from lattice QCD with exact chiral symmetry," Phys. Rev. D 78, 054502 (2008) [arXiv:0806.4744 [hep-lat]]. 
[51] K. A. Olive, M. Pospelov, Y. Z. Qian, A. Coc, M. Casse and E. Vangioni-Flam, "Constraints on the variations of the fundamental couplings," Phys. Rev. D 66, 045022 (2002) arXiv:hep-ph/0205269.

[52] J. F. Donoghue, "When Effective Field Theories Fail," arXiv:0909.0021 [hep-ph].

[53] J. F. Donoghue, B. R. Holstein and B. Borasoy, "SU(3) baryon chiral perturbation theory and long distance regularization," Phys. Rev. D 59, 036002 (1999) arXiv:hep-ph/9804281.

J. F. Donoghue and B. R. Holstein, "Improving the convergence of SU(3) baryon chiral perturbation theory," arXiv:hep-ph/9803312.

[54] M. Golterman, "Applications of chiral perturbation theory to lattice QCD," arXiv:0912.4042 [hep-lat].

[55] D. Androic et al. [G0 Collaboration], "Strange Quark Contributions to Parity-Violating Asymmetries in the Backward Angle G0 Electron Scattering Experiment," Phys. Rev. Lett. 104, 012001 (2010) arXiv:0909.5107 [nucl-ex]].

S. Baunack et al., "Measurement of Strange Quark Contributions to the Vector Form Factors of the Proton at $\mathrm{Q}^{* *} 2=0.22(\mathrm{GeV} / \mathrm{c})^{* *} 2$," Phys. Rev. Lett. 102, 151803 (2009) arXiv:0903.2733 [nucl-ex]].

A. Acha et al. [HAPPEX collaboration], "Precision Measurements of the Nucleon Strange Form Factors at $\mathrm{Q}^{* *} 2 \quad 0.1-\mathrm{GeV}^{* *} 2$," Phys. Rev. Lett. 98, 032301 (2007) arXiv:nucl-ex/0609002.

D. T. Spayde et al. [SAMPLE Collaboration], "The strange quark contribution to the proton's magnetic moment," Phys. Lett. B 583, 79 (2004) arXiv:nucl-ex/0312016].

K. S. Kumar and P. A. Souder, "Strange quarks and parity violation," Prog. Part. Nucl. Phys. 45, S333 (2000).

[56] U. G. Meißner, V. Mull, J. Speth and J. W. van Orden, "Strange vector currents and the OZI-rule," Phys. Lett. B 408, 381 (1997) arXiv:hep-ph/9701296. 